\title{
30. LATEST OLIGOCENE THROUGH EARLY MIOCENE ISOTOPIC STRATIGRAPHY AND DEEP-WATER PALEOCEANOGRAPHY OF THE WESTERN EQUATORIAL ATLANTIC: SITES 926 AND 9291
}

\author{
B.P. Flower, ${ }^{2}$ J.C. Zachos, ${ }^{2}$ and E. Martin ${ }^{3}$
}

\begin{abstract}
Stable isotopic $\left(\delta^{18} \mathrm{O}\right.$ and $\left.\delta^{13} \mathrm{C}\right)$ and strontium isotopic $\left({ }^{87} \mathrm{Sr} /{ }^{86} \mathrm{Sr}\right)$ data generated from Ocean Drilling Program (ODP) Sites 926 and 929 on Ceara Rise provide a detailed chemostratigraphy for the latest Oligocene through early Miocene of the western Equatorial Atlantic. Oxygen isotopic data based on the benthic foraminifer Cibicidoides mundulus exhibit four distinct $\delta^{18} \mathrm{O}$ excursions of more than $0.5 \%$, including event Mi1 near the Oligocene/Miocene boundary from 23.9 to 22.9 Ma and increases at about 21.5, 18 and 16.5 Ma, probably reflecting episodes of early Miocene Antarctic glaciation events (Mila, Milb, and Mi2). Carbon isotopic data exhibit well-known $\delta^{13} \mathrm{C}$ increases near the Oligocene/Miocene boundary ( 23.8 to $\left.22.6 \mathrm{Ma}\right)$ and near the early/middle Miocene boundary ( 17.5 to $16 \mathrm{Ma})$. Strontium isotopic data reveal an unconformity in the Hole 926A sequence at about 304 meters below sea floor (mbsf); no such unconformity is observed at Site 929. The age of the unconformity is estimated as 17.9 to $16.3 \mathrm{Ma}$ based on a magnetostratigraphic calibration of the ${ }^{87} \mathrm{Sr} /{ }^{86} \mathrm{Sr}$ seawater curve, and as 17.4 to 15.8 Ma based on a biostratigraphic calibration. Shipboard biostratigraphic data are more consistent with the biostratigraphic calibration.

Similar isotopic values at Sites 926 and 929 during the latest Oligocene through early Miocene suggests both were bathed by the same water mass throughout this interval. However, comparison with North Atlantic Site 608 (representing a Northern Component Water [NCW] end-member) and South Atlantic Site 704 (representing a Southern Component Water [SCW] endmember) reveals a switch in south-to-north deep-water $\delta^{13} \mathrm{C}$ gradients between about 23 and $19 \mathrm{Ma}$. These results confirm earlier suggestions that Atlantic deep-water circulation featured a single SCW source near the Oligocene/Miocene boundary and developed a second NCW source during the early Miocene.
\end{abstract}

\section{INTRODUCTION}

A major focus in Cenozoic paleoceanography is the relationship between global climate change and deep ocean circulation. An increasing number of studies has implicated deep-water circulation in climatic change on a variety of different time scales. In the late Quaternary, several studies have demonstrated a reduction or redistribution of North Atlantic Deep Water (NADW) during glacial intervals (e.g., Boyle and Keigwin, 1987; Oppo and Fairbanks, 1990; de Menocal et al., 1992). A decrease in NADW production is widely thought to be tied to Northern Hemisphere glaciations through reduction of the Boreal Heat Pump (e.g., Imbrie et al., 1992; 1993). In this way the deep circulation may play a major role in Quaternary Northern Hemisphere climates.

On longer time scales of the Cenozoic, deep circulation patterns underwent several major reorganizations, related to tectonic changes (Fig. 1). The opening of Drake Passage during the late Oligocene led to the formation of the circumpolar current, leading to the thermal isolation of Antarctica, cooling of the southern oceans, and increased formation of cold, bottom waters (e.g., Shackleton and Kennett 1975; Kennett, 1977), representing Southern Component Water (SCW). At lower latitudes, the Tethys Ocean may have been a source of warm, saline deep water during parts of the Oligocene (Kennett and Stott, 1990 ) and again during the early to early middle Miocene (Woodruff

${ }^{1}$ Shackleton, N.J., Curry, W.B., Richter, C., and Bralower, T.J. (Eds.), 1997. Proc. $O D P$, Sci. Results, 154: College Station, TX (Ocean Drilling Program).

${ }^{2}$ Institute of Marine Sciences and Earth Sciences Board, University of California, Santa Cruz; Santa Cruz, CA 95064, U.S.A. flower@earthsci.ucsc.edu U.S.A. and Savin, 1989; Flower and Kennett, 1994, 1995) until its constriction during the early middle Miocene (Yilmaz, 1993).

Several studies have suggested that SCW formation increased during Antarctic glaciations of the late Paleogene and early Neogene (e.g., Kennett, 1977; Miller and Fairbanks, 1985; Kennett and Stott, 1990; Woodruff and Savin, 1989; Wright et al., 1992; Zachos et al., 1992; Flower and Kennett, 1995). The long-term evolution of the Antarctic cryosphere during the Oligocene through middle Miocene may be closely related to the strength of SCW sources. However, at least one study (Miller et al., 1991) shows a brief pulse of Northern Component Water (NCW) in the earliest Oligocene, coincident with a period of widespread glaciation on Antarctica. Other studies have implicated NCW as a major control on Antarctic glaciation through moisture supply (Schnitker, 1980; Prentice and Matthews, 1991). As a transitional interval in the evolution of SCW and NCW sources, the late Oligocene through early Miocene provides a test of hypotheses regarding the role of deep ocean circulation in Antarctic glacial history.

\section{Early Miocene Paleoceanography}

The late Oligocene through early Miocene witnessed a series of intermittent Antarctic glaciations and was an important transitional interval in the development of the Neogene "Icehouse World." Benthic oxygen isotopic records in the late Oligocene through early Miocene show a series of long-term glacial-interglacial fluctuations that culminated in the warmest interval of the Neogene near the early/ middle Miocene boundary, followed by global cooling of the middle Miocene (Miller et al., 1987, 1991; Flower and Kennett, 1993, 1995). But the history of deep circulation prior to the establishment of a strong NCW source (such as the modern NADW) is not well known. Little is known about the early Miocene ocean in general, due in part 


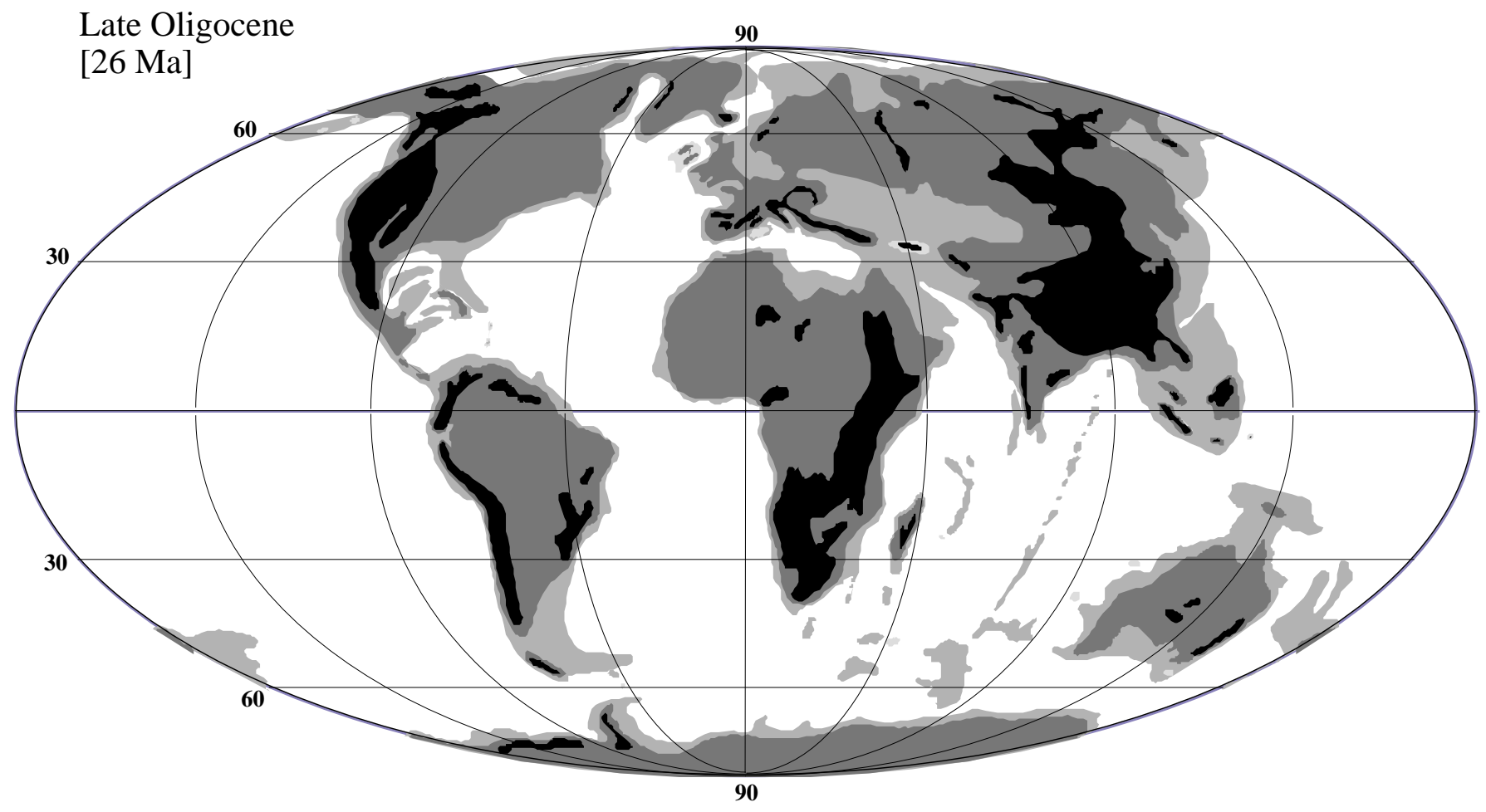

Figure 1. Paleogeographic reconstruction at $\sim 26$ Ma showing the configuration of late Oligocene continents and oceans. Black indicates mountain ranges, dark gray indicates continental areas, and light gray indicates shallow ocean areas ( $<1000 \mathrm{~m}$ water depth). Note the open Drake Passage and the shallow connections between the Atlantic, Tethys, and Indian Oceans.

to widespread unconformities in lower Miocene sections of existing deep-sea drilling holes (e.g., Wright et al., 1992; Wright and Miller, 1993).

Existing sedimentologic, faunal, and isotopic data suggest a different deep circulation during the early Miocene, without a strong Atlantic-to-Pacific flow pattern. An important change in the locus of deposition of biosilica from the North Atlantic to the Indian and $\mathrm{Pa}$ cific Oceans, termed the "Silica Switch," occurred near the early/ middle Miocene boundary (Keller and Barron, 1983; Woodruff and Savin, 1989; Barron and Baldauf, 1990). Biosilica deposition ceased over widespread areas of the North Atlantic at this time. This pattern suggests fundamental changes in either the aging of deep waters (and by inference in flow pattern and preservation) and/or in biogenic silica production. Subsidence of the Iceland Plateau during the middle Miocene may have allowed for increased production of NCW (Schnitker, 1980; Wright et al., 1992). However, carbon isotopic evidence indicates a moderate contribution from NCW sources during the early Miocene from 19.5 to $16 \mathrm{Ma}$ (Wright et al., 1992).

Additionally, the northern Indian Ocean may have been a source of warm, saline deep water during the early to early middle Miocene (Woodruff and Savin, 1989; Flower and Kennett, 1994, 1995). As both the western and eastern portals of the Tethys Ocean were still open at this time (Yilmaz, 1993), intermediate to deep waters in the western equatorial Atlantic could potentially have a Tethyan source. The Ceara Rise depth transect offers an excellent opportunity to resolve the history of Atlantic Ocean deep-water chemistry and circulation during the early Miocene. Depth transects of deep-sea sediment cores have proven effective in tracing deep-water history in the equatorial Pacific (Farrell and Prell, 1989), the Indian Ocean (Peterson and Backman, 1990), and the southwest Pacific (Flower and Kennett, 1995). A depth transect of sites cored on Ceara Rise by Ocean Drilling Program (ODP) Leg 154 (Fig. 2) allows examination of At- lantic Ocean circulation history. Carbon isotopic data on benthic foraminifers from this transect can be used to infer bottom-water chemistry, and thus to reconstruct deep-water circulation, because the $\delta^{13} \mathrm{C}$ composition of deep waters decreases as deep waters age and accumulate remineralized carbon. Variations in carbonate content can also be used to trace relative strengths of NADW and Antarctic Bottom Water $(\mathrm{AABW})$ production because of the corrosivity to carbonate of AABW. These approaches have been successfully employed in the investigation of Atlantic deep-water history during the late Quaternary (Curry et al., 1988). Now with the availability of Leg 154 cores, there is great potential for detailed bathymetric reconstructions based on five holes drilled at 3.0, 3.3, 3.6, 4.0, and $4.4 \mathrm{~km}$ (Sites 925, 927, 926, 928, and 929, respectively). Several intervals of the Oligocene through Quaternary are being studied using this depth transect.

In this study, stable isotopic $\left(\delta^{18} \mathrm{O}\right.$ and $\left.\delta^{13} \mathrm{C}\right)$ and strontium isotopic $\left({ }^{87} \mathrm{Sr} /{ }^{86} \mathrm{Sr}\right)$ data are presented for two sites in the Ceara Rise depth transect in the late Oligocene through early Miocene. Data from Sites 926 and $929(\sim 3.4 \mathrm{~km}$ and $\sim 4.2 \mathrm{~km}$ paleodepth, respectively, based on a simple thermal subsidence model) are used to (1) establish a chronostratigraphy integrating $\delta^{18} \mathrm{O}, \delta^{13} \mathrm{C}$ and ${ }^{87} \mathrm{Sr} /{ }^{86} \mathrm{Sr}$ data and (2) examine deep-water circulation during a key interval in Cenozoic climatic evolution, the upper Oligocene through lower Miocene. A companion paper (Flower et al., this volume) presents 5-k.y. resolution stable isotopic data spanning the Oligocene-Miocene boundary.

\section{METHODS}

Samples from Holes 926A, 926B and 929A were freeze-dried, weighed, soaked and shaken in deionized water, and washed over a $63-\mu \mathrm{m}$ sieve, then dried and weighed again. Individual benthic fora- 


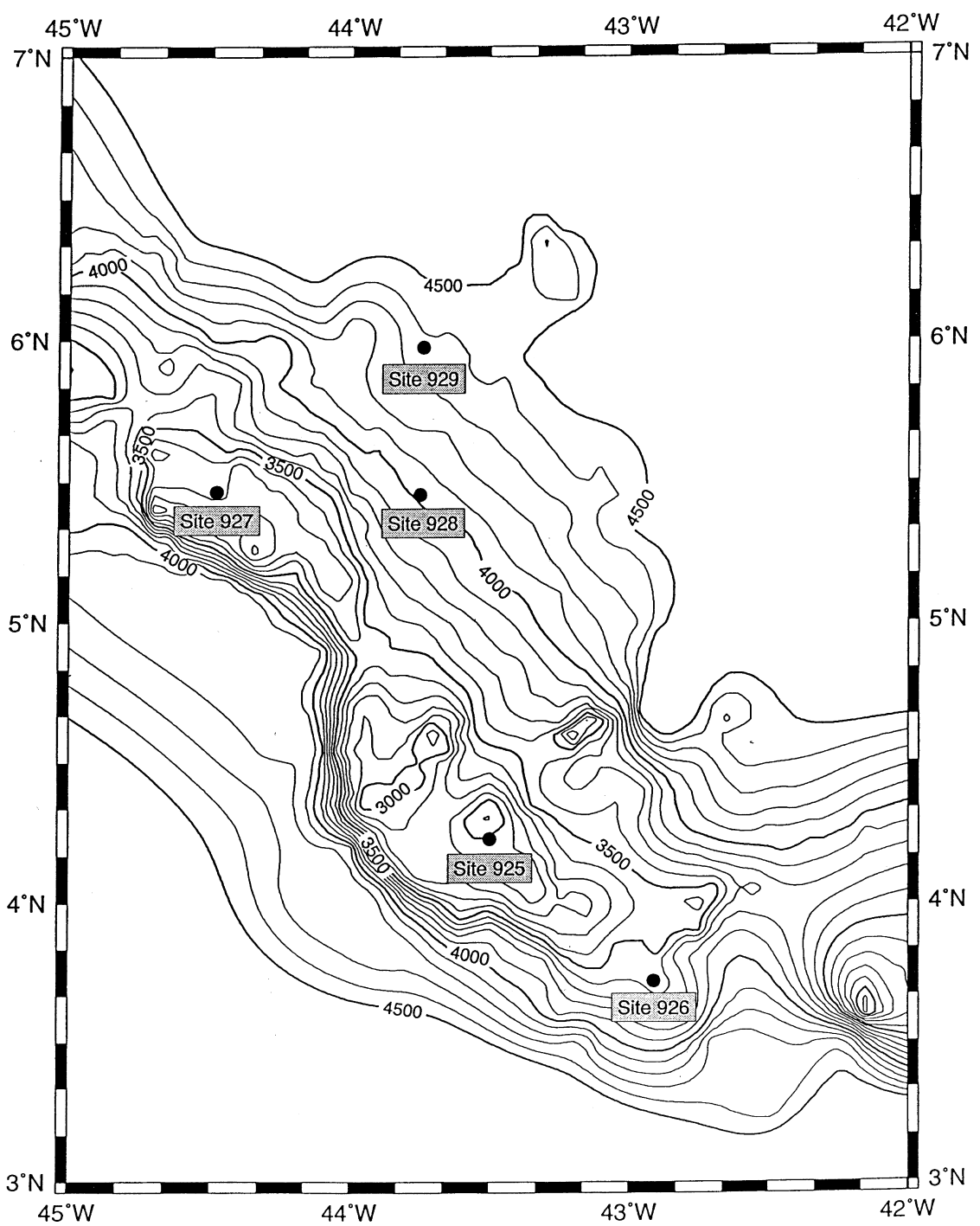

Figure 2. Location map of Leg 154 drill sites on Ceara Rise, western Equatorial Atlantic (contour interval 100 m).

minifers were hand-picked from the 150 - to $250-\mu \mathrm{m}$ size fraction for stable isotopic analysis. Approximately seven specimens of the benthic foraminifer Cibicidoides mundulus were used for each analysis. This epifaunal taxon is demonstrated to calcify close to the $\delta^{13} \mathrm{C}$ of $\Sigma \mathrm{CO}_{2}$ and with a constant offset from $\delta^{18} \mathrm{O}$ (e.g., Woodruff et al., 1980; Graham et al., 1981; Belanger et al., 1981).

Specimens for stable isotopic analysis were placed in an ultrasonic bath in methanol to remove adhering particles, roasted under vacuum at $375^{\circ} \mathrm{C}$, and reacted in orthophosphoric acid at $90^{\circ} \mathrm{C}$. The evolved $\mathrm{CO}_{2}$ gas was analyzed on-line by a Fisons Prism or Optima isotope ratio mass spectrometer equipped with an automated preparation device at the University of California, Santa Cruz. Instrumental precision based on NBS-19 and Carrera Marble carbonate standards run daily was $\pm 0.10 \%$ or for $\delta^{18} \mathrm{O}$ and $\pm 0.05 \%$ o for $\delta^{13} \mathrm{C}$. All stable isotopic data are expressed using standard $\delta$ notation in per mil relative to the Peedee belemnite (PDB) carbonate standard (Table 1).

Strontium isotopic data were generated at the University of Florida, Gainesville. For Sr isotopic analyses, approximately $0.60 \mathrm{mg}$ of planktonic foraminifers were broken open, ultrasonically cleaned to separate test fragments from any internal clay, and then reweighed. The samples were then dissolved in $1 \mathrm{ml} 2-\mathrm{N} \mathrm{HCl}$ (distilled Optima grade acid) and centrifuged. The supernatant was dried, then redissolved in $50 \mu 13.5-\mathrm{N} \mathrm{HNO}_{3}$ (Optima grade) and loaded onto small teflon columns packed with $\mathrm{Sr}$ selective crown ether resin (ElChroM Industries, Inc.). Sr was separated using a technique modified from Pin and Bassin (1992). Blanks for this technique contained $150 \mathrm{pg} \mathrm{Sr}$.

Approximately $250 \mu \mathrm{m} \mathrm{Sr}$ was then loaded with tantalum oxide onto tungsten filaments and analyzed for ${ }^{87} \mathrm{Sr} /{ }^{86} \mathrm{Sr}$ and $\mathrm{Sr}$ concentration on a VG Micromass 354 triple collector thermal ionization mass spectrometer. Two hundred ratios were collected at $2 \mathrm{~V}$. The measured ${ }^{87} \mathrm{Sr} /{ }^{86} \mathrm{Sr}$ value and the total variability for $15 \mathrm{NBS} 987$ standards run over the same time interval was $0.710244 \pm 0.000023$. This external precision represents the minimum uncertainty assigned to any sample (Table 2).

\section{RESULTS}

Site $926 \delta^{18} \mathrm{O}$ exhibits a series of $\sim 0.5 \%$ variations, which reach maxima of $1.6 \%$ o to $1.7 \%$ o from about 472 to 443 meters below sea floor (mbsf), from about 417 to $396 \mathrm{mbsf}$, and from about 368 to 356 mbsf (Fig. 3). Site $926 \delta^{13} \mathrm{C}$ exhibits values greater than $1.3 \%$ o from about 483 to $437 \mathrm{mbsf}$, and values greater than $1.6 \%$ o from about 303 to 273 mbsf.

Hole $929 \mathrm{~A} \delta^{18} \mathrm{O}$ exhibits maximum values of greater than $1.6 \%$ from about 323 to $307 \mathrm{mbsf}$, followed by lower values averaging 
Table 1. Stable isotopic data from Holes 926A, 926B, and 929A.

\begin{tabular}{|c|c|c|c|c|c|c|c|c|c|}
\hline $\begin{array}{l}\text { Core, section, } \\
\text { interval }(\mathrm{cm})\end{array}$ & $\begin{array}{l}\text { Depth } \\
\text { (mbsf) }\end{array}$ & $\begin{array}{l}\text { Depth } \\
\text { (mcd) }\end{array}$ & $\begin{array}{l}\text { Age } \\
(\mathrm{Ma})\end{array}$ & $\begin{array}{l}\delta^{13} \mathrm{C} \\
(\% o)\end{array}$ & $\begin{array}{l}\delta^{18} \mathrm{O} \\
(\%)\end{array}$ & $\begin{array}{l}\text { Core, section, } \\
\text { interval }(\mathrm{cm})\end{array}$ & $\begin{array}{l}\text { Depth } \\
\text { (mbsf) }\end{array}$ & $\begin{array}{l}\text { Depth } \\
\text { (mcd) }\end{array}$ & $\begin{array}{l}\text { Age } \\
\text { (Ma) }\end{array}$ \\
\hline 154-926A- & & & & & & $42 X-2,35-38$ & 385.95 & 415.76 & 21.23 \\
\hline $30 \mathrm{H}-2,30-35$ & 271.80 & 300.90 & 15.33 & 1.15 & 1.40 & $42 \mathrm{X}-3,35-38$ & 387.45 & 417.26 & 21.27 \\
\hline $30 \mathrm{H}-3,30-35$ & 273.30 & 302.40 & 15.38 & 1.61 & 1.01 & $42 X-4,35-38$ & 388.95 & 418.76 & 21.32 \\
\hline $30 \mathrm{H}-4,30-35$ & 274.80 & 303.90 & 15.42 & 1.60 & 0.99 & $42 X-5,35-38$ & 390.45 & 420.26 & 21.37 \\
\hline $30 \mathrm{H}-5,25-30$ & 276.25 & 305.35 & 15.47 & 1.60 & 1.35 & $42 \mathrm{X}-6,35-38$ & 391.95 & 421.76 & 21.41 \\
\hline $30 \mathrm{H}-6,30-35$ & 277.80 & 306.90 & 15.52 & 1.36 & 1.14 & $43 \mathrm{X}-1,34-39$ & 394.04 & 423.85 & 21.47 \\
\hline $30 \mathrm{H}-7,15-20$ & 279.15 & 308.25 & 15.56 & 1.57 & 1.09 & $43 \mathrm{X}-1,95-100$ & 394.65 & 424.46 & 21.49 \\
\hline $31 \mathrm{H}-1,35-38$ & 279.85 & 308.95 & 15.58 & 1.07 & 1.27 & $43 \mathrm{X}-2,34-39$ & 395.54 & 425.35 & 21.52 \\
\hline $31 \mathrm{H}-2,35-38$ & 281.35 & 310.45 & 15.63 & 1.46 & 1.39 & $43 \mathrm{X}-3,34-39$ & 397.04 & 426.85 & 21.57 \\
\hline $31 \mathrm{H}-3,35-38$ & 282.85 & 311.95 & 15.67 & 1.69 & 1.22 & $43 X-4,34-39$ & 398.54 & 428.35 & 21.61 \\
\hline $31 \mathrm{H}-3,95-98$ & 283.45 & 312.55 & 15.69 & 1.88 & 0.91 & $43 \mathrm{X}-4,95-100$ & 399.15 & 428.96 & 21.63 \\
\hline $31 \mathrm{H}-4,35-38$ & 284.35 & 313.45 & 15.72 & 1.75 & 1.04 & $43 \mathrm{X}-5,34-39$ & 400.04 & 429.85 & 21.66 \\
\hline $31 \mathrm{H}-4,100-103$ & 285.00 & 314.10 & 15.74 & 1.79 & 1.10 & $44 X-1,95-98$ & 404.35 & 434.16 & 21.79 \\
\hline $31 \mathrm{H}-5,35-38$ & 285.85 & 314.95 & 15.77 & 1.49 & 1.48 & $44 \mathrm{X}-2,34-37$ & 405.24 & 435.05 & 21.82 \\
\hline $31 \mathrm{H}-5,35-38$ & 285.85 & 314.95 & 15.77 & 1.45 & 1.50 & $44 \mathrm{X}-3,34-37$ & 406.74 & 436.55 & 21.86 \\
\hline $31 \mathrm{H}-5,95-98$ & 286.45 & 315.55 & 15.79 & 1.33 & 1.22 & $44 X-4,95-98$ & 408.85 & 438.66 & 21.93 \\
\hline $31 \mathrm{H}-6,35-38$ & 287.35 & 316.45 & 15.81 & 1.37 & 0.89 & $44 X-5,34-37$ & 409.74 & 439.55 & 21.95 \\
\hline $31 \mathrm{H}-6,95-98$ & 287.95 & 317.05 & 15.83 & 1.36 & 0.52 & $44 X-6,34-37$ & 411.24 & 441.05 & 22.00 \\
\hline $31 \mathrm{H}-7,35-38$ & 288.85 & 317.95 & 15.86 & 1.64 & 1.15 & $45 \mathrm{X}-1,95-98$ & 414.05 & 443.86 & 22.08 \\
\hline $32 \mathrm{H}-1,35-38$ & 289.35 & 317.91 & 15.86 & 1.64 & 1.01 & $45 \times-2,34-37$ & 414.94 & 444.75 & 22.11 \\
\hline $32 \mathrm{H}-1,95-98$ & 289.95 & 318.51 & 15.88 & 1.55 & 0.94 & $45 \mathrm{X}-3,34-37$ & 416.44 & 446.25 & 22.16 \\
\hline $32 \mathrm{H}-2,35-38$ & 290.85 & 319.41 & 15.91 & 1.24 & 0.75 & $45 X-4,95-98$ & 418.55 & 448.36 & 22.22 \\
\hline $32 \mathrm{H}-2,95-98$ & 291.45 & 320.01 & 15.93 & 1.67 & 1.00 & $45 \mathrm{X}-5,95-98$ & 420.05 & 449.86 & 22.27 \\
\hline $32 \mathrm{H}-3,35-38$ & 292.35 & 320.91 & 15.95 & $\begin{array}{l}1.01 \\
1.58\end{array}$ & 1.05 & $45 \mathrm{X}-6,97-100$ & 421.57 & 451.38 & 22.31 \\
\hline $32 \mathrm{H}-3,95-98$ & 292.95 & 321.51 & 15.97 & 1.41 & 1.17 & $46 \mathrm{X}-1,95-98$ & 423.75 & 453.56 & 22.38 \\
\hline $32 \mathrm{H}-4,35-38$ & 293.85 & 322.41 & 16.00 & 1.57 & 1.12 & $46 \mathrm{X}-2,95-98$ & 425.25 & 455.06 & 22.43 \\
\hline $32 \mathrm{H}-4,90-93$ & 294.40 & 322.96 & 16.02 & 1.16 & 0.88 & $46 \mathrm{X}-3,29-32$ & 426.09 & 455.9 & 22.45 \\
\hline $32 \mathrm{H}-5,35-38$ & 295.35 & 323.91 & 16.05 & 1.69 & 0.98 & $46 \mathrm{X}-4,34-38$ & 427.64 & 457.45 & 22.50 \\
\hline $32 \mathrm{H}-5,95-98$ & 295.95 & 324.51 & 16.07 & 1.53 & 1.12 & $46 \mathrm{X}-5,35-39$ & 429.15 & 458.96 & 22.54 \\
\hline $32 \mathrm{H}-6,35-38$ & 296.85 & 325.41 & $\begin{array}{l}16.07 \\
16.09\end{array}$ & 1.47 & 1.02 & $46 \mathrm{X}-6,30-33$ & 430.6 & 460.41 & 22.59 \\
\hline $32 \mathrm{H}-6,95-98$ & 297.45 & 326.01 & 16.11 & $\begin{array}{l}1.59 \\
1.59\end{array}$ & $\begin{array}{l}1.09 \\
1.09\end{array}$ & $47 \mathrm{X}-1,34-38$ & 432.74 & 462.55 & 22.65 \\
\hline $33 \mathrm{H}-1,33-37$ & 298.83 & 327.93 & $\begin{array}{l}16.11 \\
16.17\end{array}$ & 1.79 & 0.92 & $\begin{array}{l}47 \mathrm{X}-2,92-96 \\
4\end{array}$ & 434.82 & 464.63 & 22.72 \\
\hline $33 \mathrm{H}-1,95-99$ & 299.45 & 328.55 & 16.19 & 1.72 & 1.36 & $47 X-3,34-38$ & 435.74 & 465.55 & 22.75 \\
\hline $33 \mathrm{H}-2,35-39$ & 300.35 & 329.45 & 16.22 & 1.63 & 1.24 & $47 X-4,34-38$ & 437.24 & 467.05 & 22.79 \\
\hline $33 \mathrm{H}-2,95-99$ & 300.95 & 330.05 & 16.24 & 1.81 & 1.03 & 47X-5, 95-98 & 439.35 & 469.16 & 22.85 \\
\hline $33 \mathrm{H}-3,35-39$ & 301.85 & 330.95 & 16.27 & 2.14 & 1.21 & $47 X-6,34-38$ & 440.24 & 470.05 & 22.88 \\
\hline $33 \mathrm{H}-3,92-96$ & 302.42 & 331.52 & 16.29 & 1.87 & 1.26 & $48 X-1,35-37$ & 442.45 & 472.26 & 22.95 \\
\hline $33 \mathrm{H}-4,35-39$ & 303.35 & 332.45 & 17.81 & $\begin{array}{l}1.01 \\
1.08\end{array}$ & 1.17 & $48 \mathrm{X}-1,95-97$ & 443.05 & 472.86 & 22.97 \\
\hline $33 \mathrm{H}-5,35-39$ & 304.85 & 333.95 & 17.83 & $\begin{array}{l}1.08 \\
1.08\end{array}$ & 1.36 & $48 \mathrm{X}-2,35-37$ & 443.95 & 473.76 & 23.00 \\
\hline $34 \mathrm{H}-1,41-45$ & 308.41 & 337.51 & 17.90 & 1.02 & 1.00 & $48 X-3,35-37$ & 445.45 & 475.26 & 23.04 \\
\hline $34 \mathrm{H}-3,35-39$ & 311.35 & 340.45 & 17.96 & 1.31 & 1.36 & $48 \mathrm{X}-3,98-101$ & 446.08 & 475.89 & 23.06 \\
\hline $34 \mathrm{H}-4,35-39$ & 312.85 & 341.95 & 17.98 & 1.16 & 1.49 & $48 \mathrm{X}-4,35-37$ & 446.95 & 476.76 & 23.09 \\
\hline $34 \mathrm{H}-5,35-39$ & 314.35 & 343.45 & 18.01 & 1.00 & 1.51 & $48 X-4,92-95$ & 447.52 & 477.33 & 23.10 \\
\hline $34 \mathrm{H}-6,35-39$ & 315.85 & 344.95 & 18.04 & 0.90 & 1.11 & $48 X-5,35-37$ & 448.45 & 478.26 & 23.13 \\
\hline $34 \mathrm{H}-7,35-39$ & 317.35 & 346.45 & 18.07 & 0.74 & 1.52 & $48 X-5,91-94$ & 449.01 & 478.82 & 23.15 \\
\hline $35 \mathrm{H}-1,34-39$ & 317.84 & 348.15 & 18.10 & 1.16 & 1.16 & $48 X-6,35-37$ & 449.95 & 479.76 & 23.18 \\
\hline $35 \mathrm{H}-2,35-40$ & 319.35 & 349.66 & 18.17 & 1.10 & 1.29 & 48X-6, 91-94 & 450.51 & 480.32 & 23.19 \\
\hline $35 \mathrm{H}-3,35-40$ & 320.85 & 351.16 & 18.24 & 0.97 & 1.13 & $49 X-1,23-26$ & 451.93 & 481.74 & 23.24 \\
\hline $35 \mathrm{H}-4,35-40$ & 322.35 & 352.66 & 18.31 & 1.04 & 1.45 & $49 \mathrm{X}-1,92-95$ & 452.62 & 482.43 & 23.26 \\
\hline $154-926 \mathrm{~B}-$ & & & & & & $49 \mathrm{X}-2,39-42$ & 453.59 & 483.40 & 23.29 \\
\hline 154-926B- & 32325 & 35306 & & & & $49 X-2,89-92$ & 454.09 & 483.90 & 23.30 \\
\hline $35 \mathrm{X}-5,35-38$ & 323.25 & 353.06 & 18.32 & 1.11 & 1.13 & $49 \mathrm{X}-3,30-33$ & 455.00 & 484.81 & 23.33 \\
\hline $35 \mathrm{X}-5,35-38$ & 323.25 & 353.06 & 18.32 & 1.03 & 1.00 & $49 \mathrm{X}-3,97-100$ & 455.67 & 485.48 & 23.35 \\
\hline $35 \mathrm{X}-6,35-38$ & 324.75 & 354.56 & 18.39 & 0.59 & 0.93 & $49 \mathrm{X}-4,30-33$ & 456.50 & 486.31 & 23.38 \\
\hline $35 \mathrm{X}-7,35-38$ & 326.25 & 356.06 & 18.46 & 1.04 & 1.37 & $49 \mathrm{X}-4,97-100$ & 457.17 & 486.98 & 23.40 \\
\hline $36 \mathrm{X}-1,33-36$ & 326.83 & 356.64 & 18.48 & 0.89 & 1.09 & $49 \times-519-22$ & 457.89 & $\begin{array}{l}400.90 \\
48770\end{array}$ & 23.42 \\
\hline $36 \mathrm{X}-2,28-31$ & 328.28 & 358.09 & 18.55 & 1.19 & 0.92 & $\begin{array}{l}49 X-5,19-22 \\
49 X-5,93-96\end{array}$ & $\begin{array}{l}457.89 \\
458.63\end{array}$ & $\begin{array}{l}487.10 \\
488.44\end{array}$ & $\begin{array}{l}23.42 \\
2344\end{array}$ \\
\hline $36 \mathrm{X}-3,9-12$ & 329.59 & 359.40 & 18.61 & 1.13 & 1.29 & $\begin{array}{l}49 X-5,93-96 \\
49 X-6,34-37\end{array}$ & $\begin{array}{l}458.63 \\
459.54\end{array}$ & $\begin{array}{l}488.44 \\
489.35\end{array}$ & $\begin{array}{l}23.44 \\
23.47\end{array}$ \\
\hline $36 \mathrm{X}-4,16-19$ & 331.16 & 360.97 & 18.68 & 0.60 & 1.08 & $\begin{array}{l}49 \mathrm{X}-6,34-37 \\
50 \mathrm{X}-1,27-30\end{array}$ & $\begin{array}{l}461.67 \\
\end{array}$ & 491.48 & 23.52 \\
\hline $37 \mathrm{X}-1,32-37$ & 336.52 & 366.33 & 18.92 & 0.82 & 1.54 & $\begin{array}{l}50 \times-1,2-30 \\
50 \mathrm{X}-1,100-103\end{array}$ & 462.40 & 492.41 & 23.53 \\
\hline $37 \mathrm{X}-2,32-37$ & 338.02 & 367.83 & 18.99 & 0.94 & 1.01 & $\begin{array}{l}50 X-1,100-103 \\
50 X-2,38-40\end{array}$ & $\begin{array}{l}462.40 \\
463.28\end{array}$ & $\begin{array}{l}492.21 \\
493.09\end{array}$ & 23.55 \\
\hline $37 \mathrm{X}-3,32-37$ & 339.52 & 369.33 & 19.06 & 1.05 & 1.20 & $\begin{array}{l}50 \mathrm{X}-2,28-40 \\
50 \mathrm{X}-2,99-102\end{array}$ & $\begin{array}{l}403.28 \\
463.89\end{array}$ & 493.70 & 23.57 \\
\hline $37 \mathrm{X}-4,32-37$ & 341.02 & 370.83 & 19.12 & 0.91 & 1.09 & $\begin{array}{l}50 X-2,3-102 \\
50 \mathrm{X}-3,32-35\end{array}$ & $\begin{array}{l}463.89 \\
464.72\end{array}$ & 494.53 & 23.59 \\
\hline $37 \mathrm{X}-5,32-37$ & 342.52 & 372.33 & 19.19 & 1.02 & 1.17 & $\begin{array}{l}50 X-3,32-35 \\
50 X-3,98-101\end{array}$ & $\begin{array}{l}464.12 \\
465.38\end{array}$ & $\begin{array}{l}494.53 \\
495.19\end{array}$ & $\begin{array}{l}23.59 \\
23.60\end{array}$ \\
\hline $37 \mathrm{X}-5,32-37$ & 342.52 & 372.33 & 19.19 & 0.89 & 1.23 & $\begin{array}{l}50 X-3,98-101 \\
50 X-4,35-38\end{array}$ & $\begin{array}{l}465.38 \\
466.25\end{array}$ & 496.06 & 23.63 \\
\hline $38 \mathrm{X}-1,35-38$ & 346.15 & 375.96 & 19.35 & 0.53 & 1.11 & $\begin{array}{l}50 X-4,35-38 \\
50 X-4,90-93\end{array}$ & 46680 & 496.61 & 23.64 \\
\hline $38 \mathrm{X}-2,35-38$ & 347.65 & 377.46 & 19.42 & 0.88 & 0.34 & $\begin{array}{l}50 X-4,90-93 \\
50 X-5,33-36\end{array}$ & $\begin{array}{l}466.80 \\
467.73\end{array}$ & $\begin{array}{l}496.61 \\
497.54\end{array}$ & $\begin{array}{l}23.64 \\
23.66\end{array}$ \\
\hline $38 \mathrm{X}-3,30-34$ & 349.1 & 378.91 & 19.49 & 0.89 & 1.33 & $\begin{array}{l}0 \mathrm{X}-5,30-30 \\
50 \mathrm{X}-5,90-93\end{array}$ & $\begin{array}{l}468.30 \\
468.30\end{array}$ & 498.11 & 23.68 \\
\hline $38 \mathrm{X}-4,30-34$ & 350.6 & 380.41 & 19.55 & 0.89 & 1.03 & $50 \mathrm{X}-6,43-46$ & $\begin{array}{l}408.300 \\
469.33\end{array}$ & $\begin{array}{l}490.11 \\
49.14\end{array}$ & 23.71 \\
\hline $38 \mathrm{X}-5,30-34$ & 352.1 & 381.91 & 19.62 & 0.86 & 1.30 & $\begin{array}{l}0 \times-6,43-40 \\
50 \mathrm{X}-6,87-90\end{array}$ & $\begin{array}{l}469.33 \\
469.77\end{array}$ & $\begin{array}{l}499.14 \\
499.58\end{array}$ & 23.73 \\
\hline $38 \mathrm{X}-6,30-34$ & 353.6 & 383.41 & 19.69 & 0.99 & 1.17 & $\begin{array}{l}50 \mathrm{X}-6,87-90 \\
51 \mathrm{X}-1,35-38\end{array}$ & $\begin{array}{l}469.1 / 15 \\
471.35\end{array}$ & $\begin{array}{l}499.58 \\
501.16\end{array}$ & 23.78 \\
\hline $38 \mathrm{X}-7,30-34$ & 355.1 & 384.91 & 20.29 & 0.68 & 1.44 & $\begin{array}{l}11 X-1,35-38 \\
51 X-1.95-98\end{array}$ & 471.95 & 501.76 & 2380 \\
\hline $39 \mathrm{X}-1,32-35$ & 355.82 & 385.63 & 20.31 & 0.43 & 1.64 & $\begin{array}{l}51 X-1,95-98 \\
51 X-2,35-38\end{array}$ & 472.85 & 502.66 & 23.83 \\
\hline $39 \mathrm{X}-2,33-36$ & 357.33 & 387.14 & 20.36 & 0.75 & 1.21 & $\begin{array}{l}11 \mathrm{X}-2,35-38 \\
51 \mathrm{X}-2,95-98\end{array}$ & $\begin{array}{l}4 / 2.85 \\
473.45\end{array}$ & $\begin{array}{l}502.00 \\
503.26\end{array}$ & 23.85 \\
\hline $39 \mathrm{X}-3,36-39$ & 358.86 & 388.67 & 20.40 & 0.58 & 1.45 & $51 X-3,35-38$ & $\begin{array}{l}473.49 \\
474.35\end{array}$ & $\begin{array}{l}503.20 \\
504.16\end{array}$ & 23.87 \\
\hline $39 \mathrm{X}-4,36-39$ & 360.36 & 390.17 & 20.45 & 1.02 & 1.25 & $\begin{array}{l}11 X-3,35-38 \\
51 X-3,95-98\end{array}$ & 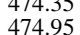 & $\begin{array}{l}504.16 \\
504.76\end{array}$ & $\begin{array}{l}23.87 \\
23.89\end{array}$ \\
\hline $39 X-5,32-35$ & 361.82 & 391.63 & 20.49 & 1.15 & 1.46 & $\begin{array}{l}51 \mathrm{X}-3,95-98 \\
51 \mathrm{X}-4,35-38\end{array}$ & 475.85 & 505.66 & 23.92 \\
\hline $40 \mathrm{X}-1,35-38$ & 365.45 & 395.26 & 20.60 & 0.84 & 1.51 & $\begin{array}{l}51 X-4,35-38 \\
51 X-4,95-98\end{array}$ & 476.45 & 506.26 & 23.94 \\
\hline $40 \mathrm{X}-2,35-38$ & 366.95 & 396.76 & 20.65 & 0.64 & 1.49 & $\begin{array}{l}51 X-4,95-98 \\
51 X-5,35-38\end{array}$ & $\begin{array}{l}4 / 6.45 \\
477.35\end{array}$ & $\begin{array}{l}506.26 \\
507.16\end{array}$ & $\begin{array}{l}23.94 \\
23.97\end{array}$ \\
\hline $40 \mathrm{X}-3,35-38$ & 368.45 & 398.26 & 20.70 & 0.51 & 1.76 & $\begin{array}{l}31 \mathrm{X}-5,35-38 \\
51 \mathrm{X}-5,95-98\end{array}$ & $\begin{array}{l}471.35 \\
477.95\end{array}$ & $\begin{array}{l}507.10 \\
507.76\end{array}$ & 23.99 \\
\hline $40 \mathrm{X}-4,35-38$ & 369.95 & 399.76 & 20.74 & 0.71 & 1.28 & $51 X-6,35-38$ & $\begin{array}{l}478.85 \\
478.85\end{array}$ & 508.66 & 24.02 \\
\hline $40 \mathrm{X}-5,35-38$ & 371.45 & 401.26 & 20.79 & 0.80 & 1.35 & $\begin{array}{l}11 X-6,35-38 \\
51 X-6,95-98\end{array}$ & 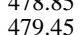 & $\begin{array}{l}508.66 \\
509.26\end{array}$ & $\begin{array}{l}24.02 \\
24.05\end{array}$ \\
\hline $40 X-6,35-38$ & 372.95 & 402.76 & 20.83 & 0.83 & 1.16 & $\begin{array}{l}31 X-6,93-98 \\
51 X-7.35-38\end{array}$ & $\begin{array}{l}4 / 9.45 \\
480.35\end{array}$ & $\begin{array}{l}509.26 \\
51016\end{array}$ & $\begin{array}{l}24.05 \\
24.10\end{array}$ \\
\hline $41 \mathrm{X}-1,35-38$ & 374.75 & 404.56 & 20.89 & 0.79 & 1.57 & $\begin{array}{l}51 X-7,35-38 \\
52 \times-1,35-38\end{array}$ & $\begin{array}{l}480.35 \\
480.85\end{array}$ & $\begin{array}{l}510.16 \\
510.66\end{array}$ & 24.12 \\
\hline $41 \mathrm{X}-3,35-38$ & 377.25 & 407.06 & 20.96 & 0.81 & 1.06 & $\begin{array}{l}52 X-1,35-38 \\
52 X-1,95-98\end{array}$ & $\begin{array}{l}480.85 \\
481.45\end{array}$ & $\begin{array}{l}510.66 \\
511.26\end{array}$ & 24.15 \\
\hline $41 X-4,35-38$ & 378.75 & 408.56 & 21.01 & 0.53 & 1.13 & $\begin{array}{l}52 \mathrm{X}-1,95-98 \\
52 \mathrm{X}-2,35-38\end{array}$ & $\begin{array}{l}481.45 \\
482.35\end{array}$ & $\begin{array}{l}5112.26 \\
512.16\end{array}$ & $\begin{array}{l}24.15 \\
24.19\end{array}$ \\
\hline $41 X-5,35-38$ & 380.25 & 410.06 & 21.05 & 0.43 & 1.60 & $\begin{array}{l}212-2,30-38 \\
52 X-2,95-98\end{array}$ & $\begin{array}{l}482.3 \mathrm{~J} \\
482.95\end{array}$ & 512.76 & $\begin{array}{l}24.19 \\
24.21\end{array}$ \\
\hline $42 X-1,35-38$ & 384.45 & 414.26 & 21.18 & 0.64 & 1.56 & $\begin{array}{l}22 \mathrm{X}-2,95-98 \\
52 \mathrm{X}-3,35-38\end{array}$ & 483.85 & 513.66 & 24.25 \\
\hline
\end{tabular}


Table 1 (continued).

\begin{tabular}{|c|c|c|c|c|c|}
\hline $\begin{array}{l}\text { Core, section, } \\
\text { interval }(\mathrm{cm})\end{array}$ & $\begin{array}{l}\text { Depth } \\
\text { (mbsf) }\end{array}$ & $\begin{array}{l}\text { Depth } \\
\text { (mcd) }\end{array}$ & $\begin{array}{l}\text { Age } \\
\text { (Ma) }\end{array}$ & $\begin{array}{l}\delta^{13} \mathrm{C} \\
(\% o)\end{array}$ & $\begin{array}{l}\delta^{18} \mathrm{O} \\
(\% o)\end{array}$ \\
\hline $52 X-3,95-98$ & 484.45 & 514.26 & 24.27 & 0.80 & 0.83 \\
\hline $52 X-4,35-38$ & 485.35 & 515.16 & 24.31 & 0.61 & 0.82 \\
\hline $52 X-4,95-98$ & 485.95 & 515.76 & 24.33 & 0.63 & 1.14 \\
\hline $52 X-5,35-38$ & 486.85 & 516.66 & 24.37 & 0.57 & 0.80 \\
\hline $52 X-5,95-98$ & 487.45 & 517.26 & 24.39 & 0.70 & 0.93 \\
\hline $52 X-6,35-38$ & 488.35 & 518.16 & 24.43 & 0.71 & 1.11 \\
\hline $52 X-6,95-98$ & 488.95 & 518.76 & 24.45 & 0.98 & 0.87 \\
\hline $52 \mathrm{X}-7,35-38$ & 489.85 & 519.66 & 24.49 & 0.84 & 0.89 \\
\hline $53 X-1,36-40$ & 490.56 & 520.37 & 24.51 & 0.85 & 1.09 \\
\hline $53 \mathrm{X}-1,95-99$ & 491.15 & 520.96 & 24.54 & 0.68 & 0.89 \\
\hline $53 \mathrm{X}-2,35-39$ & 492.05 & 521.86 & 24.57 & 0.71 & 0.70 \\
\hline $53 X-2,95-99$ & 492.65 & 522.46 & 24.60 & 0.66 & 0.74 \\
\hline $53 \mathrm{X}-3,34-39$ & 493.54 & 523.35 & 24.63 & 0.38 & 1.09 \\
\hline $53 \mathrm{X}-3,108-112$ & 494.28 & 524.09 & 24.66 & 0.55 & 0.90 \\
\hline $53 X-4,32-37$ & 495.02 & 524.83 & 24.69 & 0.70 & 0.96 \\
\hline $53 \mathrm{X}-4,102-106$ & 495.72 & 525.53 & 24.72 & 0.48 & 1.04 \\
\hline $53 X-5,34-39$ & 496.54 & 526.35 & 24.76 & 0.65 & 0.85 \\
\hline $53 \mathrm{X}-5,95-99$ & 497.15 & 526.96 & 24.78 & 0.66 & 0.96 \\
\hline $53 X-6,35-40$ & 498.05 & 527.86 & 24.82 & 0.65 & 1.30 \\
\hline $53 \mathrm{X}-6,95-99$ & 498.65 & 528.46 & 24.84 & 0.67 & 1.12 \\
\hline \multicolumn{6}{|l|}{ 154-929A- } \\
\hline $25 \mathrm{X}-1,35-39$ & 224.35 & 239.77 & 15.12 & 1.13 & 1.10 \\
\hline $25 \mathrm{X}-1,90-95$ & 224.90 & 240.32 & 15.29 & 1.36 & 1.19 \\
\hline $25 \mathrm{X}-2,35-39$ & 225.85 & 241.27 & 15.56 & 1.13 & 1.13 \\
\hline $25 \mathrm{X}-2,95-100$ & 226.45 & 241.87 & 15.74 & 1.24 & 1.06 \\
\hline $25 X-3,35-39$ & 227.35 & 242.77 & 15.88 & 1.13 & 1.15 \\
\hline $25 \mathrm{X}-3,95-100$ & 227.95 & 243.37 & 15.94 & 1.38 & 1.44 \\
\hline $25 X-4,36-39$ & 228.86 & 244.28 & 16.04 & 1.46 & 1.35 \\
\hline $25 \mathrm{X}-4,101-105$ & 229.51 & 244.93 & 16.11 & 1.78 & 1.33 \\
\hline $25 \mathrm{X}-5,35-39$ & 230.35 & 245.77 & 16.20 & 1.40 & 0.98 \\
\hline $25 X-5,96-101$ & 230.96 & 246.38 & 16.26 & 1.47 & 1.41 \\
\hline $25 \mathrm{X}-6,33-37$ & 231.83 & 247.25 & 16.36 & 1.35 & 0.91 \\
\hline $25 \mathrm{X}-6,122-126$ & 232.72 & 248.14 & 16.45 & 1.61 & 1.63 \\
\hline $25 \mathrm{X}-7,15-23$ & 233.15 & 248.57 & 16.50 & 1.67 & 1.19 \\
\hline $26 \mathrm{X}-1,121-124$ & 234.81 & 250.23 & 16.68 & 1.9 & 1.47 \\
\hline $26 \mathrm{X}-1,37-40$ & 233.97 & 249.39 & 16.59 & 1.97 & 1.34 \\
\hline $26 \mathrm{X}-2,121-124$ & 236.31 & 251.73 & 16.84 & 1.68 & 1.04 \\
\hline $26 \mathrm{X}-2,27-30$ & 235.37 & 250.79 & 16.74 & 1.35 & 0.83 \\
\hline $26 \mathrm{X}-3,121-124$ & 237.81 & 253.23 & 17.00 & 1.61 & 1.11 \\
\hline $26 \mathrm{X}-3,41-44$ & 237.01 & 252.43 & 16.92 & 1.29 & 0.95 \\
\hline $26 \mathrm{X}-4,121-124$ & 239.31 & 254.73 & 17.16 & 1.41 & 1.37 \\
\hline $26 \mathrm{X}-4,41-44$ & 238.51 & 253.93 & 17.08 & 1.2 & 1.02 \\
\hline $26 \mathrm{X}-5,121-124$ & 240.81 & 256.23 & 17.33 & 1.21 & 1.22 \\
\hline $26 \mathrm{X}-5,41-44$ & 240.01 & 255.43 & 17.24 & 1.1 & 1.05 \\
\hline $26 X-6,41-44$ & 241.51 & 256.93 & 17.40 & 1.01 & 1.37 \\
\hline $27 \mathrm{X}-1,31-34$ & 243.71 & 259.13 & 17.64 & 1.05 & 1.18 \\
\hline $27 \mathrm{X}-1,95-98$ & 244.35 & 259.77 & 17.71 & 1.01 & 1.17 \\
\hline $27 \mathrm{X}-2,30-33$ & 245.20 & 260.62 & 17.80 & 0.95 & 1.45 \\
\hline $27 \mathrm{X}-2,94-97$ & 245.84 & 261.26 & 17.87 & 0.83 & 0.81 \\
\hline $27 \mathrm{X}-3,32-35$ & 246.72 & 262.14 & 17.96 & 0.73 & 1.27 \\
\hline $27 X-3,94-97$ & 247.34 & 262.76 & 18.03 & 0.83 & 1.63 \\
\hline $27 X-4,30-33$ & 248.20 & 263.62 & 18.12 & 0.97 & 1.27 \\
\hline $27 \mathrm{X}-4,94-97$ & 248.84 & 264.26 & 18.19 & 0.82 & 0.96 \\
\hline $27 X-5,30-33$ & 249.70 & 265.12 & 18.28 & 0.89 & 1.44 \\
\hline $27 X-5,94-97$ & 250.34 & 265.76 & 18.35 & 0.66 & 1.34 \\
\hline $27 X-6,30-33$ & 251.20 & 266.62 & 18.45 & 0.87 & 1.23 \\
\hline 27X-6, 94-97 & 251.84 & 267.26 & 18.54 & 0.46 & 1.01 \\
\hline $28 \mathrm{X}-1,35-38$ & 253.35 & 268.77 & 18.73 & 0.86 & 1.23 \\
\hline $28 \mathrm{X}-1,110-113$ & 254.10 & 269.52 & 18.83 & 0.75 & 1.32 \\
\hline $28 \mathrm{X}-2,35-38$ & 254.85 & 270.27 & 18.92 & 0.63 & 1.15 \\
\hline $28 \mathrm{X}-2,110-113$ & 255.60 & 271.02 & 19.02 & 0.69 & 1.33 \\
\hline $28 \mathrm{X}-3,35-38$ & 256.35 & 271.77 & 19.12 & 0.61 & 1.10 \\
\hline $28 \mathrm{X}-3,110-113$ & 257.10 & 272.52 & 19.21 & 0.91 & 0.70 \\
\hline $28 \mathrm{X}-4,35-38$ & 257.85 & 273.27 & 19.31 & 0.70 & 1.20 \\
\hline $28 \mathrm{X}-4,110-113$ & 258.60 & 274.02 & 19.41 & 0.90 & 1.02 \\
\hline $28 \mathrm{X}-5,35-38$ & 259.35 & 274.77 & 19.50 & 0.72 & 1.19 \\
\hline $29 \mathrm{X}-1,35-38$ & 262.55 & 277.97 & 19.92 & 0.89 & 1.32 \\
\hline
\end{tabular}

about $1.2 \%$ or the remainder of the lower Miocene (Fig. 4). Hole 929A $\delta^{13} \mathrm{C}$ exhibits values greater than $1.3 \%$ o from about 332 to 323 mbsf, and values greater than $1.6 \%$ ofrom about 238 to $229 \mathrm{mbsf}$.

\section{DISCUSSION}

Sediment lithology in Holes 926A, 926B, and 929A is primarily a moderately indurated, clayey nannofossil chalk. Although some evidence exists for bulk carbonate diagenesis based on sediment lithification (Shipboard Scientific Party, 1995a; 1995b) and major element ratios $(\mathrm{Sr} / \mathrm{Ca}, \mathrm{Mg} / \mathrm{Ca})$ of pore waters and bulk carbonates (Delaney

\begin{tabular}{|c|c|c|c|c|c|}
\hline $\begin{array}{l}\text { Core, section, } \\
\text { interval }(\mathrm{cm})\end{array}$ & $\begin{array}{l}\text { Depth } \\
\text { (mbsf) }\end{array}$ & $\begin{array}{l}\text { Depth } \\
\text { (mcd) }\end{array}$ & $\begin{array}{l}\text { Age } \\
(\mathrm{Ma})\end{array}$ & $\begin{array}{l}\delta^{13} \mathrm{C} \\
(\% o)\end{array}$ & $\begin{array}{l}\delta^{18} \mathrm{O} \\
(\%)\end{array}$ \\
\hline $29 X-1,95-98$ & 263.15 & 278.57 & 20.00 & 0.72 & 1.46 \\
\hline $29 X-2,35-38$ & 264.05 & 279.47 & 20.11 & 0.99 & 1.08 \\
\hline $29 X-2,95-98$ & 264.65 & 280.07 & 20.19 & 0.84 & 1.05 \\
\hline $29 X-3,20-23$ & 265.40 & 280.82 & 20.29 & 0.43 & 1.34 \\
\hline $29 \mathrm{X}-4,35-38$ & 267.05 & 282.47 & 20.50 & 1.06 & 1.42 \\
\hline $29 X-5,35-38$ & 268.55 & 283.97 & 20.69 & 0.60 & 1.28 \\
\hline $29 X-6,35-38$ & 270.05 & 285.47 & 20.89 & 0.81 & 1.17 \\
\hline $29 X-7,35-38$ & 271.55 & 286.97 & 21.08 & 0.79 & 1.46 \\
\hline $30 X-3,35-40$ & 275.25 & 290.67 & 21.56 & 0.71 & 0.97 \\
\hline $30 X-4,35-40$ & 276.75 & 292.17 & 21.65 & 1.01 & 1.16 \\
\hline $30 X-5,35-40$ & 278.25 & 293.67 & 21.72 & 0.94 & 1.34 \\
\hline $30 X-6,35-40$ & 279.75 & 295.17 & 21.78 & 0.54 & 1.05 \\
\hline $30 X-7,35-40$ & 281.25 & 296.67 & 21.85 & 0.65 & 1.27 \\
\hline $31 X-2,35-38$ & 283.45 & 298.87 & 21.95 & 0.94 & 1.35 \\
\hline $31 X-3,35-38$ & 284.95 & 300.37 & 22.01 & 1.09 & 1.03 \\
\hline $31 X-4,35-38$ & 286.45 & 301.87 & 22.08 & 0.99 & 1.38 \\
\hline $32 X-1,33-36$ & 291.63 & 307.05 & 22.31 & 1.13 & 1.27 \\
\hline $32 \times-3,33-36$ & 294.63 & 310.05 & 22.44 & 0.85 & 1.28 \\
\hline $32 X-4,33-36$ & 296.13 & 311.55 & 22.50 & 0.97 & 1.08 \\
\hline $32 X-5,28-31$ & 297.58 & 313.00 & 22.57 & 0.63 & 1.14 \\
\hline $32 X-6,28-31$ & 299.08 & 314.50 & 22.63 & 1.01 & 1.14 \\
\hline $33 \mathrm{X}-1,52-55$ & 301.52 & 316.94 & 22.74 & 1.16 & 1.08 \\
\hline $33 X-2,52-55$ & 303.02 & 318.44 & 22.81 & 1.40 & 0.86 \\
\hline $33 \mathrm{X}-3,52-55$ & 304.52 & 319.94 & 22.87 & 0.95 & 1.24 \\
\hline $33 X-4,53-56$ & 306.03 & 321.45 & 22.94 & 1.16 & 1.28 \\
\hline $33 X-5,52-55$ & 307.52 & 322.94 & 23.00 & 1.37 & 1.68 \\
\hline $33 X-6,52-55$ & 309.02 & 324.44 & 23.07 & 1.42 & 1.63 \\
\hline $34 \mathrm{X}-1,35-39$ & 311.05 & 326.47 & 23.16 & 0.97 & 0.87 \\
\hline $34 \mathrm{X}-2,35-39$ & 312.55 & 327.97 & 23.22 & 1.48 & 1.08 \\
\hline $34 \mathrm{X}-3,35-39$ & 314.05 & 329.47 & 23.29 & 1.32 & 0.98 \\
\hline $34 \mathrm{X}-3,105-109$ & 314.75 & 330.17 & 23.32 & 1.32 & 1.73 \\
\hline $34 \mathrm{X}-4,35-39$ & 315.55 & 330.97 & 23.36 & 1.06 & 1.21 \\
\hline $34 \mathrm{X}-4,105-109$ & 316.25 & 331.67 & 23.39 & 1.24 & 1.11 \\
\hline $34 X-5,35-39$ & 317.05 & 332.47 & 23.42 & 1.27 & 1.04 \\
\hline $34 \mathrm{X}-5,105-109$ & 317.75 & 333.17 & 23.45 & 1.26 & 1.19 \\
\hline $34 X-6,35-39$ & 318.55 & 333.97 & 23.49 & 0.96 & 1.11 \\
\hline $34 \mathrm{X}-6,105-109$ & 319.25 & 334.67 & 23.52 & 1.13 & 1.75 \\
\hline $34 X-7,18-22$ & 319.88 & 335.30 & 23.55 & 0.84 & 1.17 \\
\hline $35 \mathrm{X}-1,35-39$ & 320.75 & 336.17 & 23.59 & 1.47 & 0.93 \\
\hline $35 \mathrm{X}-1,105-109$ & 321.45 & 336.87 & 23.62 & 1.05 & 1.16 \\
\hline $35 \times-2,35-39$ & 322.25 & 337.67 & 23.65 & 1.35 & 1.65 \\
\hline $35 X-2,105-109$ & 322.95 & 338.37 & 23.68 & 1.43 & 1.75 \\
\hline $35 \mathrm{X}-3,35-39$ & 323.75 & 339.17 & 23.72 & 1.28 & 1.57 \\
\hline $35 X-3,105-109$ & 324.45 & 339.87 & 23.76 & 1.05 & 1.39 \\
\hline $35 \mathrm{X}-4,35-39$ & 325.25 & 340.67 & 23.81 & 1.12 & 1.42 \\
\hline $35 \mathrm{X}-4,105-109$ & 325.95 & 341.37 & 23.85 & 0.74 & 1.22 \\
\hline $35 \times-5,35-39$ & 326.75 & 342.17 & 23.89 & 0.53 & 0.99 \\
\hline $35 X-5,105-109$ & 327.45 & 342.87 & 23.93 & 0.72 & 1.14 \\
\hline $35 X-6,35-39$ & 328.25 & 343.67 & 23.98 & 0.62 & 0.27 \\
\hline $35 \mathrm{X}-6,105-109$ & 328.95 & 344.37 & 24.02 & 1.11 & 0.92 \\
\hline $35 \mathrm{X}-7,19-23$ & 329.59 & 345.01 & 24.05 & 1.14 & 1.14 \\
\hline $36 \mathrm{X}-1,35-38$ & 330.35 & 345.77 & 24.09 & 1.35 & 0.88 \\
\hline $36 \mathrm{X}-1,105-108$ & 331.05 & 346.47 & 24.13 & 1.31 & 0.95 \\
\hline $36 \mathrm{X}-2,35-38$ & 331.85 & 347.27 & 24.18 & 1.17 & 0.94 \\
\hline $36 X-3,35-38$ & 333.35 & 348.77 & 24.26 & 0.77 & 0.83 \\
\hline $36 X-3,105-108$ & 334.05 & 349.47 & 24.30 & 0.51 & 0.49 \\
\hline $36 \mathrm{X}-4,35-38$ & 334.85 & 350.27 & 24.35 & 0.68 & 0.60 \\
\hline $36 \mathrm{X}-4,105-108$ & 335.55 & 350.97 & 24.39 & 0.77 & 0.87 \\
\hline $36 \mathrm{X}-5,35-38$ & 336.35 & 351.77 & 24.43 & 0.98 & 0.96 \\
\hline $36 X-6,35-38$ & 337.85 & 353.27 & 24.52 & 0.83 & 1.18 \\
\hline $36 \mathrm{X}-7,35-38$ & 339.35 & 354.77 & 24.60 & 0.60 & 0.92 \\
\hline $37 X-1,33-37$ & 340.03 & 355.45 & 24.64 & 0.40 & 0.74 \\
\hline $37 X-2,33-37$ & 341.53 & 356.95 & 24.72 & 0.67 & 0.90 \\
\hline $37 \mathrm{X}-3,31-35$ & 343.01 & 358.43 & 24.81 & 0.79 & 1.11 \\
\hline $37 X-4,33-37$ & 344.53 & 359.95 & 24.89 & 0.86 & 1.15 \\
\hline $37 X-5,33-37$ & 346.03 & 361.45 & 24.98 & 0.87 & 1.38 \\
\hline $37 \mathrm{X}-7,16-20$ & 348.86 & 364.28 & 25.14 & 0.58 & 0.96 \\
\hline
\end{tabular}

and Anderson, this volume; Hampt and Delaney, this volume), benthic foraminifers are well preserved. Visual inspection of washed specimens with the light microscope reveals no calcite overgrowths. In addition, our isotopic data exhibit values in the expected range. Also, thinner-walled planktonic foraminifers that are more sensitive to recrystallization yield $\delta^{18} \mathrm{O}$ and $\delta^{13} \mathrm{C}$ values within the range of expected values for the early Miocene tropical Atlantic surface ocean (B.P. Flower and J.C. Zachos, unpubl. data). Further, Sr concentrations in planktonic foraminifers (Table 2) suggest minimal recrystallization of foraminifers in the cores studied. Still, the possibility of diagenetic alteration requires that caution be exercised in interpreting small differences in isotopic values. 
Table 2. Strontium isotopic data from Holes 926A, 926B, and 929A.

\begin{tabular}{|c|c|c|c|c|c|c|c|c|}
\hline $\begin{array}{l}\text { Core, section, } \\
\text { interval }(\mathrm{cm})\end{array}$ & $\begin{array}{l}\text { Depth } \\
\text { (mbsf) }\end{array}$ & $\begin{array}{l}\text { Depth } \\
\text { (mcd) }\end{array}$ & $\begin{array}{c}\text { Age } \\
(\mathrm{Ma}, \text { biostratigraphic })\end{array}$ & ${ }^{87} \mathrm{Sr} /{ }^{86} \mathrm{Sr}$ & $2 \sigma\left(\times 10^{6}\right)$ & $\mathrm{Sr}(\mathrm{ppm})$ & $\begin{array}{c}\text { Age } \\
\text { (Ma, OMFW94) }\end{array}$ & $\begin{array}{c}\text { Age } \\
\text { (Ma, HW94) }\end{array}$ \\
\hline $\begin{array}{r}154-926 \mathrm{~A}- \\
30 \mathrm{H}-2,30-35 \\
30 \mathrm{H}-5,25-30 \\
31 \mathrm{H}-2,35-38 \\
31 \mathrm{H}-2,35-38 \\
31 \mathrm{H}-5,35-38 \\
32 \mathrm{H}-2,35-38 \\
32 \mathrm{H}-5,35-38 \\
32 \mathrm{H}-5,35-38 \\
33 \mathrm{H}-2,35-39 \\
33 \mathrm{H}-5,35-39 \\
34 \mathrm{H}-2,35-39 \\
34 \mathrm{H}-5,35-39 \\
35 \mathrm{H}-2,35-40 \\
35 \mathrm{H}-5,35-40\end{array}$ & $\begin{array}{l}271.80 \\
276.25 \\
281.35 \\
281.35 \\
285.85 \\
290.85 \\
295.35 \\
295.35 \\
300.35 \\
304.85 \\
309.85 \\
314.35 \\
319.35 \\
323.50\end{array}$ & $\begin{array}{l}300.90 \\
305.35 \\
310.45 \\
310.45 \\
314.95 \\
319.41 \\
323.91 \\
323.91 \\
329.45 \\
333.95 \\
339.55 \\
343.45 \\
349.66 \\
353.86\end{array}$ & $\begin{array}{l}14.15 \\
14.52 \\
14.95 \\
14.95 \\
15.33 \\
15.70 \\
16.08 \\
16.08 \\
16.54 \\
16.92 \\
17.39 \\
17.71 \\
18.17 \\
18.36\end{array}$ & $\begin{array}{l}0.708809 \\
0.708780 \\
0.708769 \\
0.708786 \\
0.708768 \\
0.708753 \\
0.708762 \\
0.708744 \\
0.708758 \\
0.708617 \\
0.708594 \\
0.708576 \\
0.708578 \\
0.708562\end{array}$ & $\begin{array}{l}23 \\
23 \\
23 \\
23 \\
23 \\
23 \\
23 \\
23 \\
23 \\
23 \\
23 \\
23 \\
23 \\
23\end{array}$ & $\begin{array}{l}864 \\
862 \\
888 \\
888 \\
984 \\
866 \\
809 \\
825 \\
827 \\
920 \\
971 \\
881 \\
896 \\
913\end{array}$ & $\begin{array}{l}15.3 \\
15.7 \\
15.8 \\
\\
15.9 \\
16.0 \\
16.0 \\
\\
16.2 \\
18.1 \\
18.5 \\
18.7 \\
18.7 \\
18.9\end{array}$ & $\begin{array}{l}11.8 \\
14.5 \\
14.9 \\
\\
13.0 \\
15.3 \\
15.1 \\
\\
15.4 \\
17.4 \\
16.9 \\
17.9 \\
17.9 \\
18.1\end{array}$ \\
\hline $\begin{array}{l}154-926 \mathrm{~B}- \\
35 \mathrm{X}-5,35-38 \\
36 \mathrm{X}-2,28-31 \\
36 \mathrm{X}-4,16-19 \\
37 \mathrm{X}-2,32-37 \\
37 \mathrm{X}-5,32-37 \\
38 \mathrm{X}-2,35-38 \\
38 \mathrm{X}-5,30-34 \\
39 \mathrm{X}-2,33-36 \\
39 \mathrm{X}-5,32-35 \\
40 \mathrm{X}-2,35-38 \\
40 \mathrm{X}-5,35-38 \\
41 \mathrm{X}-3,35-38 \\
41 \mathrm{X}-5,35-38 \\
42 \mathrm{X}-5,35-38 \\
43 \mathrm{X}-5,34-39 \\
44 \mathrm{X}-5,34-37 \\
45 \mathrm{X}-5,34-37 \\
46 \mathrm{X}-3,29-32 \\
47 \mathrm{X}-5,35-39 \\
47 \mathrm{X}-5,35-39 \\
48 \mathrm{X}-5,35-37 \\
49 \mathrm{X}-5,19-22 \\
50 \mathrm{X}-2,38-40\end{array}$ & $\begin{array}{l}323.25 \\
328.28 \\
331.16 \\
338.02 \\
342.52 \\
347.65 \\
352.10 \\
357.33 \\
361.82 \\
366.95 \\
371.45 \\
377.25 \\
380.25 \\
390.45 \\
400.04 \\
409.74 \\
419.44 \\
426.09 \\
438.75 \\
438.75 \\
448.45 \\
457.89 \\
463.28\end{array}$ & $\begin{array}{l}353.06 \\
358.09 \\
360.97 \\
367.83 \\
372.33 \\
377.46 \\
381.91 \\
387.14 \\
391.63 \\
396.76 \\
401.26 \\
407.06 \\
410.06 \\
420.26 \\
430.46 \\
439.55 \\
449.25 \\
455.90 \\
468.56 \\
468.56 \\
478.26 \\
487.70 \\
493.09\end{array}$ & $\begin{array}{l}18.32 \\
18.55 \\
18.68 \\
18.99 \\
19.19 \\
19.42 \\
19.62 \\
19.82 \\
19.98 \\
20.17 \\
20.33 \\
20.54 \\
20.64 \\
21.01 \\
21.37 \\
21.70 \\
22.04 \\
22.28 \\
22.73 \\
22.73 \\
23.08 \\
23.42 \\
23.61\end{array}$ & $\begin{array}{l}0.708550 \\
0.708530 \\
0.708562 \\
0.708506 \\
0.708491 \\
0.708506 \\
0.708476 \\
0.708468 \\
0.708468 \\
0.708451 \\
0.708462 \\
0.708421 \\
0.708432 \\
0.708385 \\
0.708338 \\
0.708342 \\
0.708334 \\
0.708324 \\
0.708281 \\
0.708260 \\
0.708241 \\
0.708222 \\
0.708218\end{array}$ & $\begin{array}{l}23 \\
23 \\
23 \\
23 \\
23 \\
23 \\
23 \\
23 \\
23 \\
23 \\
23 \\
23 \\
23 \\
23 \\
23 \\
23 \\
23 \\
23 \\
23 \\
23 \\
23 \\
23 \\
23\end{array}$ & $\begin{array}{r}1066 \\
1000 \\
920 \\
940 \\
1041 \\
1112 \\
911 \\
975 \\
821 \\
932 \\
738 \\
861 \\
991 \\
842 \\
909 \\
884 \\
881 \\
925 \\
927 \\
868 \\
867 \\
780 \\
1966\end{array}$ & $\begin{array}{l}19.1 \\
19.4 \\
18.9 \\
19.7 \\
20.0 \\
19.7 \\
20.2 \\
20.3 \\
20.3 \\
20.6 \\
20.4 \\
21.0 \\
20.8 \\
21.5 \\
22.2 \\
22.1 \\
22.3 \\
22.4 \\
23.7 \\
24.3 \\
24.9 \\
25.0\end{array}$ & $\begin{array}{l}18.3 \\
18.6 \\
18.1 \\
18.7 \\
18.9 \\
18.7 \\
19.2 \\
19.2 \\
19.2 \\
19.5 \\
19.3 \\
20.0 \\
19.8 \\
20.7 \\
21.6 \\
21.5 \\
21.6 \\
21.8 \\
22.5 \\
23.0 \\
23.5 \\
23.5\end{array}$ \\
\hline $\begin{array}{l}\text { 154-929A- } \\
25 X-2,35-39 \\
25 X-5,35-39 \\
25 X-5,35-39 \\
26 X-3,41-44 \\
26 X-5,41-44 \\
27 X-3,32-35 \\
27 X-5,30-33 \\
28 X-1,35-38 \\
28 X-3,35-38 \\
28 X-5,35-38 \\
29 X-3,20-23 \\
30 X-1,98-103 \\
30 X-5,35-40 \\
31 X-3,35-38 \\
32 X-4,33-36 \\
33 X-5,52-55 \\
33 X-5,52-55 \\
34 X-6,35-39 \\
35 X-6,35-39 \\
36 X-6,35-38 \\
37 X-6,33-37 \\
38 X-5,30-35\end{array}$ & $\begin{array}{l}225.85 \\
230.35 \\
230.35 \\
237.01 \\
240.01 \\
246.72 \\
249.70 \\
253.35 \\
256.35 \\
259.35 \\
265.40 \\
272.88 \\
278.25 \\
284.95 \\
296.13 \\
307.52 \\
307.52 \\
318.55 \\
328.25 \\
337.85 \\
347.53 \\
355.70\end{array}$ & $\begin{array}{l}241.27 \\
245.77 \\
245.77 \\
252.43 \\
255.43 \\
262.14 \\
265.12 \\
268.77 \\
271.77 \\
274.77 \\
280.82 \\
288.30 \\
293.67 \\
300.37 \\
311.55 \\
322.94 \\
322.94 \\
333.97 \\
343.67 \\
353.27 \\
362.95 \\
371.12\end{array}$ & $\begin{array}{l}15.56 \\
16.20 \\
16.20 \\
16.92 \\
17.24 \\
17.96 \\
18.28 \\
18.73 \\
19.12 \\
19.50 \\
20.29 \\
21.25 \\
21.72 \\
22.01 \\
22.50 \\
23.00 \\
23.00 \\
23.49 \\
23.98 \\
24.52 \\
25.06 \\
25.52\end{array}$ & $\begin{array}{l}0.708772 \\
0.708753 \\
0.708680 \\
0.708705 \\
0.708653 \\
0.708569 \\
0.708564 \\
0.708553 \\
0.708526 \\
0.708560 \\
0.708397 \\
0.708466 \\
0.708438 \\
0.708363 \\
0.708295 \\
0.708277 \\
0.708246 \\
0.708240 \\
0.708206 \\
0.708190 \\
0.708157 \\
0.708116\end{array}$ & $\begin{array}{l}23 \\
23 \\
23 \\
23 \\
23 \\
23 \\
23 \\
23 \\
23 \\
23 \\
23 \\
23 \\
23 \\
23 \\
23 \\
23 \\
23 \\
23 \\
23 \\
23 \\
23 \\
23\end{array}$ & $\begin{array}{r}925 \\
844 \\
887 \\
888 \\
838 \\
841 \\
886 \\
873 \\
832 \\
824 \\
1041 \\
867 \\
786 \\
901 \\
837 \\
875 \\
836 \\
838 \\
847 \\
863 \\
1000 \\
846\end{array}$ & $\begin{array}{l}15.9 \\
16.1 \\
17.0 \\
17.6 \\
18.8 \\
18.9 \\
19.1 \\
19.5 \\
19.0 \\
21.3 \\
20.3 \\
20.7 \\
21.8 \\
23.5 \\
23.8 \\
24.5 \\
25.2 \\
25.5 \\
26.1 \\
26.9\end{array}$ & $\begin{array}{l}15.0 \\
15.5 \\
16.6 \\
17.1 \\
18.0 \\
18.1 \\
18.2 \\
18.5 \\
18.2 \\
20.4 \\
19.3 \\
19.7 \\
21.1 \\
22.2 \\
22.9 \\
23.5 \\
24.4 \\
24.5 \\
24.6 \\
25.8\end{array}$ \\
\hline
\end{tabular}

Notes: A minimum external precision of 0.000023 has been assigned to all samples, equivalent to the total range of standard values measured during this study. Ages calculated based on a magnetostratigraphic calibration (OMFW94); mimimum error is $\pm 0.61 \mathrm{~m} . \mathrm{y}$. (Oslick et al., 1994). Ages calculated based on a biostratigraphic calibration (HW94); best theoretical error is approximately \pm 0.3 m.y. (Hodell and Woodruff, 1994).

\section{Continuity and Chronology}

Recovery was nearly $100 \%$ for Holes $926 \mathrm{~A}, 926 \mathrm{~B}$, and 929A in the upper Oligocene through lower Miocene, and no turbidites or slumps were found at Site 926. Several turbidites were found at Site 929 , but these features are thin (10-cm scale) and their locations are known (Shipboard Scientific Party, 1995b). Turbidites and slumps were meticulously identified and catalogued on board ship and were avoided during sampling.

Magnetostratigraphy was inconclusive at these sites. For chronostratigraphy, we rely on biostratigraphy and strontium isotopic stratigraphy (Fig. 5). Biostratigraphy is based mainly on calcareous nannofossils and planktonic foraminifers (Shipboard Scientific Par- ty, 1995a; 1995b). The Leg 154 time scale (Curry, Shackleton, Richter, et al., 1995), based on the recent time scale of Cande and Kent (1992), was adopted here. The Cande and Kent time scales (1992; 1995) differ minimally (<40 k.y.) in the 25 - to 15 -Ma interval. Recent efforts have significantly improved the calibration of upper Oligocene through lower Miocene $\delta^{18} \mathrm{O}, \delta^{13} \mathrm{C}$, and $\mathrm{Sr}$ isotope stratigraphies to the geomagnetic polarity time scale (Miller et al., 1991; Woodruff and Savin, 1991; Wright et al., 1992; Hodell and Woodruff, 1994; Oslick et al., 1994). In the early Miocene in particular, the rapid rate of change in seawater $\mathrm{Sr}$ isotope ratios permits stratigraphic resolution of \pm 0.61 m.y. (Oslick et al., 1994).

Strontium isotopic stratigraphy reveals an unconformity in the Site 926 sequence (Fig. 5) unrecognized with shipboard work. The 


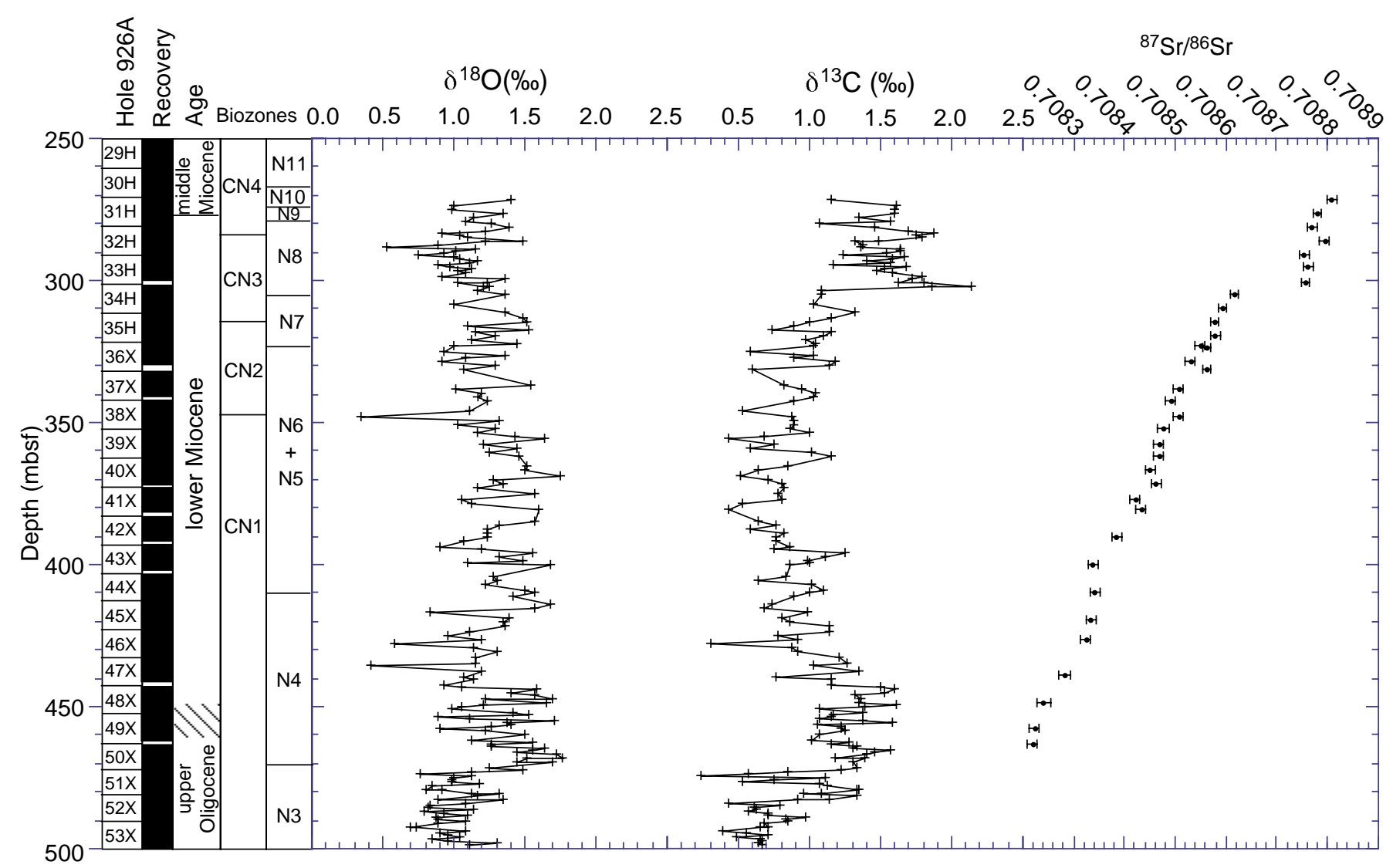

Figure 3. Upper Oligocene through lower Miocene isotopic stratigraphy $\left(\delta^{18} \mathrm{O}, \delta^{13} \mathrm{C}\right.$, and $\left.{ }^{87} \mathrm{Sr} /{ }^{86} \mathrm{Sr}\right)$ for Site 926 , plotted vs. depth in mbsf. Core recovery is shown in black, with calcareous nannoplankton and planktonic foraminifer zones. Stable isotopic analyses based on Cibicidoides mundulus. Note the oxygen isotopic increase (Mi1) near the Oligocene/Miocene boundary at about $470 \mathrm{mbsf}$, a corresponding maximum in $\delta^{13} \mathrm{C}$, and the rapid initiation of the Monterey Carbon Isotope Excursion (CM3 of Woodruff and Savin, 1991) at about 304 mbsf.

rapidity of the $\delta^{13} \mathrm{C}$ increase at Site 926 as compared with Site 929 also suggests missing section, and the unconformity is accordingly placed at 304 mbsf (Fig. 6). Based on linear regressions of ${ }^{87} \mathrm{Sr} /{ }^{86} \mathrm{Sr}$ data vs. paleomagnetically derived ages at Site 747 (Oslick et al., 1994), the age is estimated to be $\sim 17.9$ to $16.3 \mathrm{Ma}$. By comparison, the biostratigraphically calibrated ${ }^{87} \mathrm{Sr} /{ }^{86} \mathrm{Sr}$ regression of Hodell and Woodruff (1994) yields a slightly younger age of $\sim 17.4$ to $15.8 \mathrm{Ma}$. The reason for the differences between these calibrations is unknown, and may include interlaboratory differences, differential diagenesis at the sites studied, and/or errors in age assignments (Hodell and Woodruff, 1994; Oslick et al., 1994).

As shown in Figure 5, shipboard biostratigraphic data offers a test of these age calibrations of the strontium isotopic composition of seawater. Biostratigraphic datums, calibrated to the geomagnetic polarity time scale (GPTS) (Berggren et al., 1995), are compared with strontium isotopic ages calculated by magnetostratigraphy (Oslick et al., 1994) and by biostratigraphy (Hodell and Woodruff, 1994). The shipboard biostratigraphic data are more consistent with the ages calculated using the biostratigraphic calibration. In addition, the placement of event Mi1 (23.9 Ma; Miller et al., 1991) at each site is more consistent with the biostratigraphic calibration. Two important exceptions are the first occurrence (FO) of Sphenolithus belemnos in Hole 926B, and the last occurrence (LO) of Paragloborotalia kugleri in Hole 929A, which are both more consistent with the magnetostratigraphically calibrated ages. Still, this comparison suggests that the biostratigraphic calibration of the strontium isotopic composition of seawater is currently better calibrated to the GPTS, which may point to a resolution of a problem in strontium isotope stratigraphy for the early Miocene. However, this issue needs to be addressed with other paleomagnetically calibrated strontium isotopic data.

\section{Upper Oligocene-Lower Miocene Stratigraphy}

Benthic stable isotopic records from Sites 926 and 929 ( 3.4 and $\sim 4.2 \mathrm{~km}$ paleodepth, respectively) establish moderate-resolution ( $\sim 10$ to 100 k.y.) isotopic stratigraphies (Fig. 6) for the upper Oligocene (calcareous nannoplankton Zones CP19b/CN1 and planktonic foraminifer Zone P22) to the middle Miocene (calcareous nannoplankton zone CN4 and planktonic foraminifer zone N11). The oxygen and carbon isotopic values are similar to those expected for the early Miocene interval in the deep Atlantic $\left(0.5 \%\right.$ o $-1.7 \%$ of $\delta^{18} \mathrm{O}$, $0.5 \%$ o $-2.0 \%$ o for $\delta^{13} \mathrm{C}$; Miller et al., 1987, 1991; Wright et al., 1992). The $\delta^{18} \mathrm{O}$ data show an expected amplitude $(\sim 0.5 \%)$, consistent with smaller Antarctic ice sheet volumes than during the late Neogene. Oxygen isotopic maximum Mi1 ( 23.9; Miller et al., 1991) is clearly observed in both Sites 926 and 929 (Fig. 7). Three moderate $\delta^{18} \mathrm{O}$ increases of $\sim 0.5 \%$ occurred at about $21.5,18$, and $16.5 \mathrm{Ma}$ (Fig. 6), which probably correspond to events Mila, Milb, and Mi2, although higher-resolution data are needed to substantiate the correlation.

The $\delta^{13} \mathrm{C}$ records for both sites show values greater than $1.3 \%$ o from about 24 to $23.8 \mathrm{Ma}$, which represents the well-known $\delta^{13} \mathrm{C}$ maximum near the Oligocene/Miocene boundary (CM O/M of Hodell and Woodruff, 1994) associated with $\delta^{18} \mathrm{O}$ event Mi1. The $\delta^{13} \mathrm{C}$ records also exhibit maximum values greater than $1.6 \%$ in the 


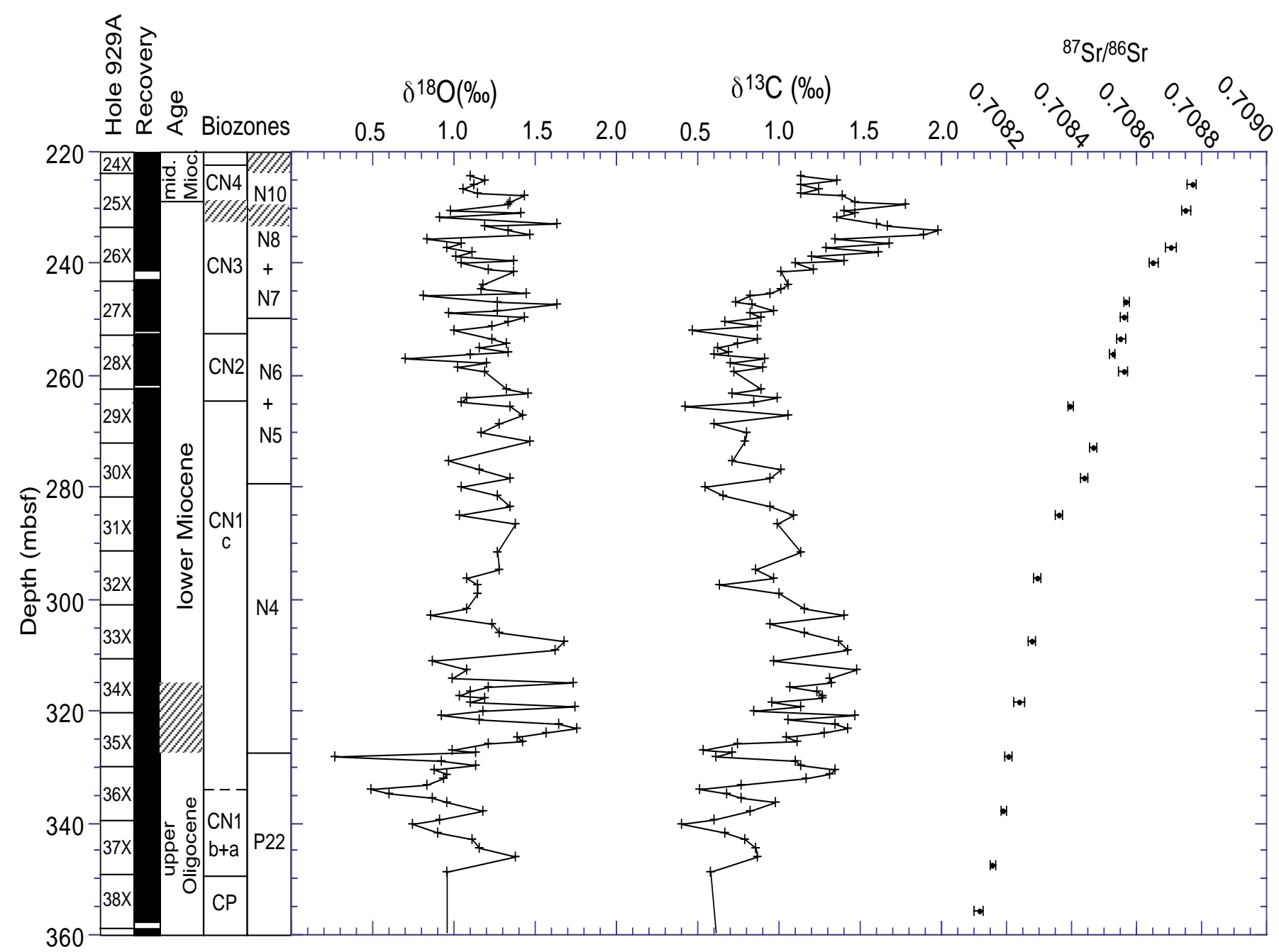

Figure 4. Upper Oligocene through lower Miocene isotopic stratigraphy $\left(\delta^{18} \mathrm{O}, \delta^{13} \mathrm{C}\right.$, and $\left.{ }^{87} \mathrm{Sr} /{ }^{86} \mathrm{Sr}\right)$ for Site 929 , plotted vs. depth in mbsf. Core recovery is shown in black, with calcareous nannoplankton and planktonic foraminifer zones. Stable isotopic analyses based on Cibicidoides mundulus. Note the oxygen isotopic increase (Mi1) near the Oligocene/Miocene boundary at about $325 \mathrm{mbsf}$, a corresponding maximum in $\delta^{13} \mathrm{C}$, and the Monterey Carbon Isotope Excursion (CM3 of Woodruff and Savin, 1991) beginning at about $240 \mathrm{mbsf}$.

Figure 5. Age vs. depth in mbsf of biostratigraphic datum levels (Shipboard Scientific Party, 1995a; 1995b) and strontium isotopic age control points employed in this study, for Sites 926 and 929. Circles indicate strontium isotope ages, open circles represent the magnetostratigraphic calibration of Oslick et al. (1994), and solid circles represent the biostratigraphic calibration of Hodell and Woodruff (1994). Minimum uncertainty of strontium isotope ages is $\pm 0.61 \mathrm{Ma}$ (Oslick et al., 1994). Crosses indicate biostratigraphic datum levels, and crosses surrounded by squares indicate biostratigraphic datum levels employed in age model. Triangle indicates Mi1. Note that shipboard biostratigraphic data are more consistent with the biostratigraphic calibration.

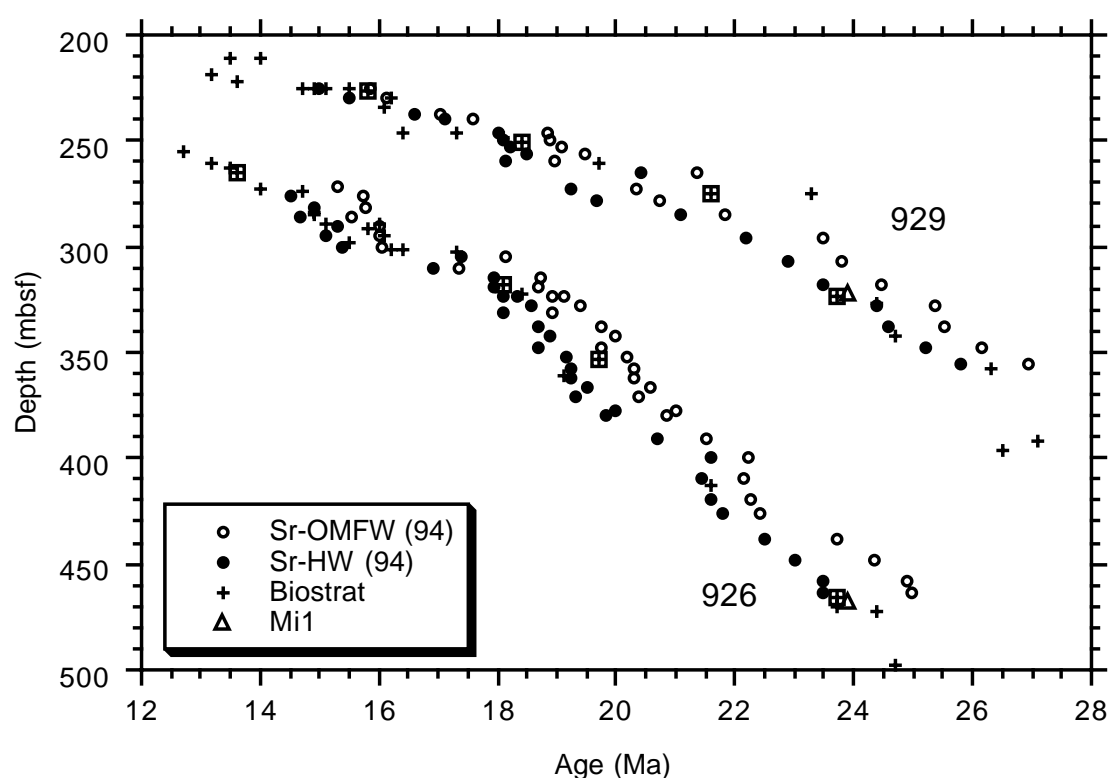




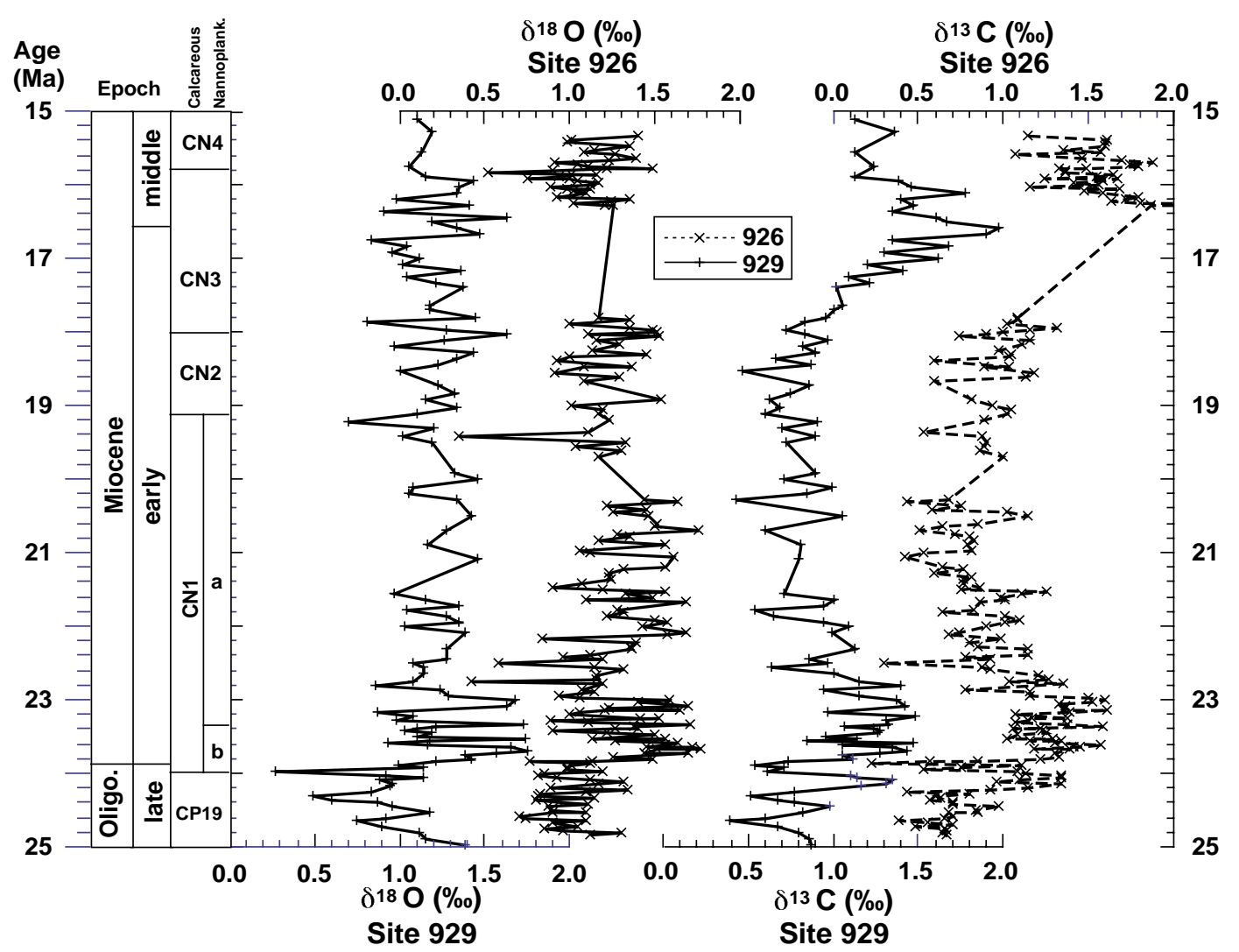

Figure 6. Late Oligocene through early Miocene oxygen and carbon isotopic records (based on Cibicidoides mundulus) for Sites 926 and 929 , plotted vs. age (Ma).

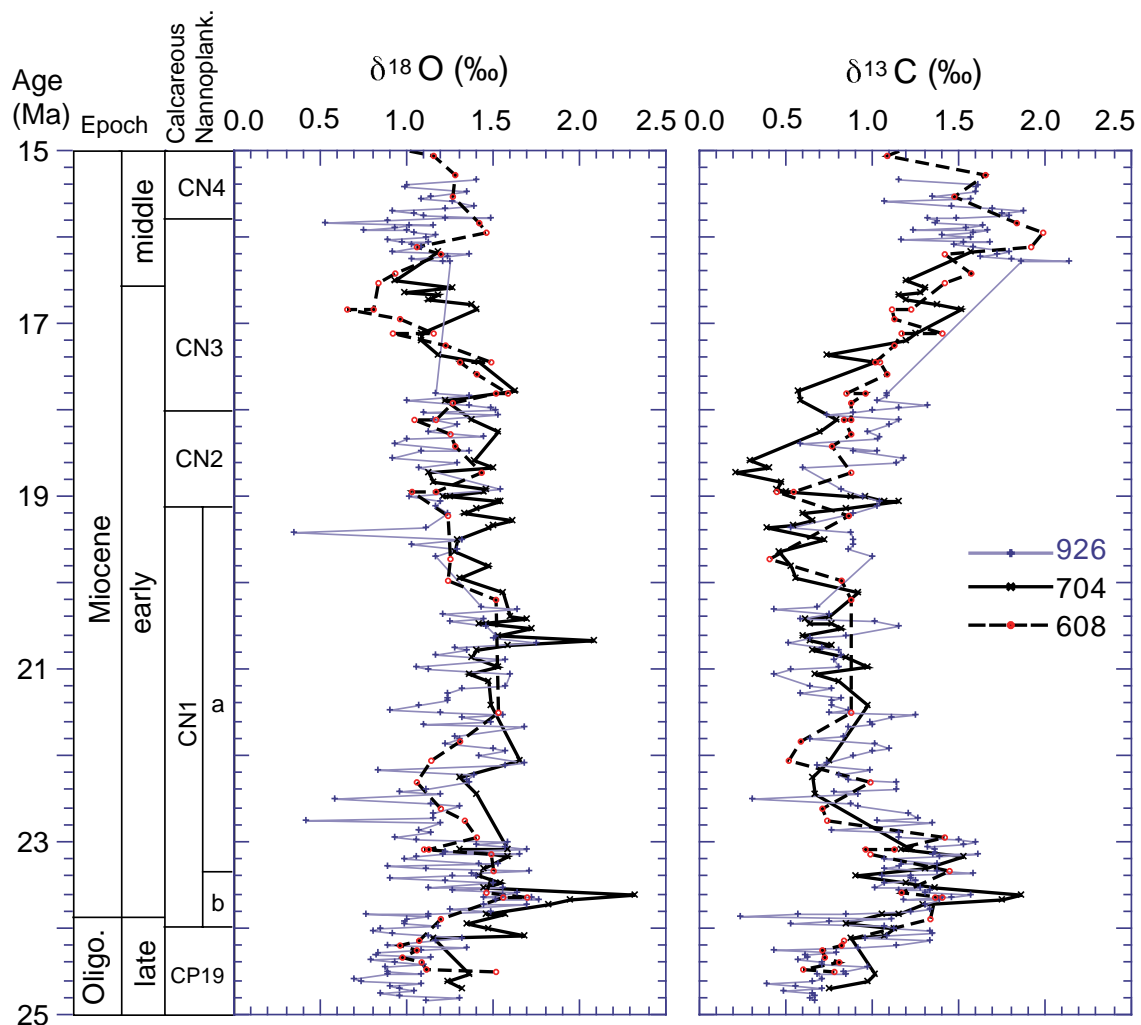

Figure 7. Composite of benthic carbon isotopic records (based on Cibicidoides), plotted vs. age for the late Oligocene through early Miocene. Site 926 record is from this study; Site 608 (Cape Basin, Northeast Atlantic) and Site 704 (Meteor Rise, South Atlantic) records are from Wright et al. (1992). 
late early Miocene from about 17.5 to $16 \mathrm{Ma}$ (Fig. 6), which probably corresponds to the early part of the well-known Monterey Carbon Isotopic Excursion (Vincent and Berger, 1985), and to $\delta^{13} \mathrm{C}$ maximum 3 of Woodruff and Savin (1991).

\section{Bathymetric and Geographic Gradients}

Comparison of isotopic gradients necessitates proper correlation of time-equivalent levels. Biostratigraphy and strontium isotopic stratigraphy allow good correlation between sites. Based on this integrated biostratigraphy and chemostratigraphy, we compare $\delta^{18} \mathrm{O}$ and $\delta^{13} \mathrm{C}$ records on a common time scale to assess gradients vs. water depth. We also compare $\delta^{13} \mathrm{C}$ values in Sites 926 and 929 with published records (Wright et al., 1992) from NCW and SCW end-member monitors Site 608 (Cape Basin, Northeast Atlantic) and Site 704 (Meteor Rise, South Atlantic).

Sites 926 and 929 have similar benthic $\delta^{18} \mathrm{O}$ values for most of the $25-15$ Ma interval (Fig. 6). Resolving any water column $\delta^{18} \mathrm{O}$ gradient changes awaits higher-resolution data. Existing carbon isotopic data also reveal no significant changes in water column $\delta^{13} \mathrm{C}$ gradients during the 25-15 Ma interval (Fig. 6). This observation suggests both sites ( $\sim 3.4$ and $\sim 4.2 \mathrm{~km}$ paleodepth, respectively) were bathed by the same water mass throughout the interval studied, in contrast to the modern ocean.

Site $926 \delta^{13} \mathrm{C}$ values are compared to a NCW end-member (Site 608) and a SCW end-member (Site 704) in Figure 7. Site 608 and 704 records were correlated to Site 926 by $\delta^{18} \mathrm{O}$ and $\delta^{13} \mathrm{C}$ stratigraphy. Any changes in intersite gradients may be controlled by the direction of deep-water flow, as the global $\delta^{13} \mathrm{C}$ signal is normalized by this comparison. Site $704 \delta^{13} \mathrm{C}$ is significantly higher than at the other sites during a brief interval near the Oligocene/Miocene boundary at $23.7 \mathrm{Ma}$, (by $\sim 0.3 \%$, based on comparison to high-resolution 929 data; Flower et al., this volume). Site 704 values are lower than at the other sites after about $19 \mathrm{Ma}$; uncertainty in correlation makes it difficult to assess meridional gradients between 23 and $19 \mathrm{Ma}$.

To the extent that existing data from Sites 608 and 704 represent end-member deep-water compositions during the early Miocene, the high $\delta^{13} \mathrm{C}$ values at Site 704 near the Oligocene/Miocene boundary suggest deep-water flow was south-to-north at this time (Fig. 7), confirming earlier work (Wright et al., 1992). In contrast, low values at Site 704 after $\sim 19$ Ma may indicate deep-water flow was north to south, implying a significant NCW source. This result confirms the suggestion based on Atlantic-Pacific $\delta^{13} \mathrm{C}$ contrasts that NCW was a component of the deep-water circulation from $\sim 19$ to $16 \mathrm{Ma}$ (Wright et al., 1992). Thus, changing meridional gradients in deep-water $\delta^{13} \mathrm{C}$ confirm that a transition in deep-water circulation from dominantly SCW sources to both SCW and NCW sources occurred between $\sim 23$ and $19 \mathrm{Ma}$.

\section{CONCLUSIONS}

Stable isotopic data based on the benthic foraminifer Cibicidoides mundulus from Sites 926 and 929 on the Ceara Rise provide $\delta^{18} \mathrm{O}$ and $\delta^{13} \mathrm{C}$ records of western Equatorial Atlantic deep waters during the latest Oligocene through early Miocene. Oxygen isotopic data exhibit four distinct $\delta^{18} \mathrm{O}$ increases of $\sim 0.5 \%$, including event Mi1 (Miller et al., 1991) near the Oligocene/Miocene boundary at $\sim 23.9$ Ma and two increases at about 21.5, 18 and 16.5 Ma, probably reflecting episodes of early Miocene Antarctic glaciation.

Carbon isotopic data exhibit increases of about $1.0 \%$ onear the Oligocene/Miocene boundary and during the late early Miocene from about 17 to $16 \mathrm{Ma}$. Carbon isotopic values reached during the latter increase (the early part of the Monterey Carbon Isotopic Excursion; CM3 of Woodruff and Savin, 1991) are the highest of the early Miocene $(\sim 2.0 \%$ o).
Together with strontium isotopic data, the data reveal an unconformity in the Hole 926A sequence at about 304 mbsf; no such unconformity is observed at Site 929 . The age of the unconformity is estimated as 17.9 to 16.3 Ma based on a magnetostratigraphic calibration of the ${ }^{87} \mathrm{Sr} /{ }^{86} \mathrm{Sr}$ seawater curve, and as 17.4 to $15.8 \mathrm{Ma}$ based on a biostratigraphic calibration. Shipboard biostratigraphic data for Sites 926 and 929 are more consistent with the biostratigraphic calibration, which may help resolve a paradox in strontium isotope stratigraphy.

Similar water column gradients in $\delta^{18} \mathrm{O}$ and $\delta^{13} \mathrm{C}$ at Sites 926 and 929 during the latest Oligocene through early Miocene suggests that both sites were bathed by the same water mass throughout this interval. However, comparison with Sites 608 (Cape Basin, Northeast Atlantic) and 704 (Meteor Rise, South Atlantic) reveals a switch in south-to-north deep-water $\delta^{13} \mathrm{C}$ gradients between about 23 and 19 Ma. These results confirm earlier suggestions that Atlantic deep-water circulation featured a single SCW source near the Oligocene/Miocene boundary and developed a second NCW source during the early Miocene.

\section{ACKNOWLEDGMENTS}

This work was supported by the JOI/USSSP (154-208396 to J.C.Z) along with partial support from NSF (OPP-9423485 to B.P.F. and OCE-9458367 to J.C.Z). We thank ODP for providing samples, T. Bralower, J. Compton, and D. Pak for thorough reviews, N. Shackleton for discussions, and Todd Green, Peter Heitz, and Kathy Vencill for laboratory assistance.

\section{REFERENCES}

Barron, J.A., and Baldauf, J.G., 1990. Development of biosiliceous sedimentation in the North Pacific during the Miocene and Early Pliocene. In Tsuchi, R. (Ed.), Pacific Neogene Events: Their Timing, Nature and Interrelationship: Tokyo (Univ. of Tokyo Press), 43-63.

Belanger, P.E., Curry, W.B., and Matthews, R.K., 1981. Core-top evaluation of benthic foraminiferal isotopic ratios for paleoceanographic interpretations. Palaeogeogr., Palaeoclimatol., Palaeoecol., 33:205-220.

Berggren, W.A., Kent, D.V., Swisher, C.C., III, and Aubry, M.-P., 1995. A revised Cenozoic geochronology and chronostratigraphy. In Berggren, W.A., Kent, D.V., and Hardenbol, J. (Eds.), Geochronology, Time Scales and Global Stratigraphic Correlation: A Unified Temporal Framework for an Historical Geology. Spec. Publ.-Soc. Econ. Paleontol. Mineral., 54.

Boyle, E.A., and Keigwin, L.D., 1987. North Atlantic thermohaline circulation during the past 20,000 years linked to high-latitude surface temperature. Nature, 330:35-40.

Cande, S.C., and Kent, D.V., 1992. A new geomagnetic polarity time scale for the Late Cretaceous and Cenozoic. J. Geophys. Res., 97:1391713951.

1995. Revised calibration of the geomagnetic polarity timescale for the Late Cretaceous and Cenozoic. J. Geophys. Res., 100:6093-6095.

Curry, W.B., Duplessy, J.C., Labeyrie, L.D., and Shackleton, N.J., 1988. Changes in the distribution of $\delta^{13} \mathrm{C}$ of glacial deep water $\Sigma \mathrm{CO}_{2}$ between the last glacial and the Holocene. Paleoceanography, 3:317-341.

Curry, W.B., Shackleton, N.J., Richter, C., et al., 1995. Proc. ODP, Init. Repts., 154: College Station, TX (Ocean Drilling Program).

de Menocal, P.B., Oppo, D.W., Fairbanks, R.G., and Prell, W.L., 1992. A 1.2 Myr record of mid-depth $\delta^{13} \mathrm{C}$ variability in the North Atlantic: relationships between climate, ocean circulation and atmospheric $\mathrm{CO}_{2}$. Paleoceanography, 7:229-250.

Farrell, J.W., and Prell, W.L., 1989. Climatic change and $\mathrm{CaCO}_{3}$ preservation: an 800,000 year bathymetric reconstruction from the central equatorial Pacific Ocean. Paleoceanography, 4:447-466.

Flower, B.P., and Kennett, J. P., 1994. The middle Miocene climatic transition: East Antarctic ice sheet development, deep ocean circulation, and global carbon cycling. Palaeogeogr., Palaeoclimatol., Palaeoecol., 108:537-555. 
1995. Middle Miocene deepwater paleoceanography in the southwest Pacific: relations with East Antarctic ice sheet development. Paleoceanography, 10:1095-1113.

Graham, D.W., Corliss, B.H., Bender, M.L., and Keigwin, L.D., Jr., 1981. Carbon and oxygen isotopic disequilibria of Recent deep-sea benthic foraminifera. Mar. Micropaleontol., 6:483-497.

Hodell, D.A., and Woodruff, 1994. Variations in the strontium isotopic ratio of seawater during the Miocene: stratigraphic and geochemical implications. Paleoceanography, 9:405-426.

Imbrie, J., Berger, A., Boyle, E., Clemens, S., Duffy, A., Howard, W., Kukla, G., Kutzbach, J., Martinson, D., McIntyre, A., Mix, A., Molfino, B., Morley, J., Peterson, L., Pisias, N., Prell, W., Raymo, M., Shackleton, N., and Toggweiler, J., 1993. On the structure and origin of major glaciation cycles, 2. The 100,000-year cycle. Paleoceanography, 8:699-735.

Imbrie, J., Boyle, E.A., Clemens, S.C., Duffy, A., Howard, W.R., Kukla, G., Kutzbach, J., Martinson, D.G., McIntyre, A., Mix, A.C., Molfino, B., Morley, J.J., Peterson, L.C., Pisias, N.G., Prell, W.L., Raymo, M.E., Shackleton, N.J., and Toggweiler, J.R., 1992. On the structure and origin of major glaciation cycles, 1. Linear responses to Milankovitch forcing. Paleoceanography, 7:701-738.

Keller, G., and Barron, J.A., 1983. Paleoceanographic implications of Miocene deep-sea hiatuses. Geol. Soc. Am. Bull., 94:590-613.

Kennett, J.P., 1977. Cenozoic evolution of Antarctic glaciation, the circumAntarctic Ocean, and their impact on global paleoceanography. J. Geophys. Res., 82:3843-3860.

Kennett, J.P., and Stott, L.D., 1990. Proteus and Proto-oceanus: ancestral Paleogene oceans as revealed from Antarctic stable isotopic results: ODP Leg 113. In Barker, P.F., Kennett, J.P., et al., Proc. ODP, Sci. Results, 113: College Station, TX (Ocean Drilling Program), 865-880.

Miller, K.G., and Fairbanks, R.G., 1985. Oligocene to Miocene carbon isotope cycles and abyssal circulation changes. In Sundquist, E.J., and Broecker, W.S. (Eds.), The Carbon Cycle and Atmospheric $\mathrm{CO}_{2}$ : Natural Variations Archean to Present. Geophys. Monogr., Am. Geophys. Union, 32:469-486.

Miller, K.G., Fairbanks, R.G., and Mountain, G.S., 1987. Tertiary oxygen isotope synthesis, sea-level history, and continental margin erosion. Paleoceanography, 2:1-19.

Miller, K.G., Wright, J.D., and Fairbanks, R.G., 1991. Unlocking the Ice House: Oligocene-Miocene oxygen isotopes, eustasy, and margin erosion. J. Geophys. Res., 96:6829-6848.

Oppo, D.W., and Fairbanks, R.G., 1990. Atlantic Ocean thermohaline circulation of the last 150,000 years: relationship of climate and atmospheric $\mathrm{CO}_{2}$. Paleoceanography, 5:277-288.

Oslick, J.S., Miller, K.G., Feigenson, M.D., and Wright, J.D., 1994. Oligocene-Miocene strontium isotopes: correlation to a glacioeustatic record. Paleoceanography, 9:427-443.

Peterson, L.C., and Backman, J., 1990. Late Cenozoic carbonate accumulation and the history of the carbonate compensation depth in the Western Equatorial Indian ocean. In Duncan, R.A., Backman, J., Peterson, L.C., et al., Proc. ODP, Sci. Results, 115: College Station (Ocean Drilling Program), 467-507.

Pin, N.C., and Bassin, C., 1992. Evaluation of a strontium-specific extraction chromatographic method for isotopic analysis in geological materials. Anal. Chim. Acta, 269:249-255.
Prentice, M.L., and Matthews, R.K., 1991. Tertiary ice sheet dynamics: the snow gun hypothesis. J. Geophys. Res., 96:6811-6827.

Schnitker, D., 1980. North Atlantic oceanography as possible cause of Antarctic glaciation and eutrophication. Nature, 284:615-616.

Shackleton, N.J., and Kennett, J.P., 1975. Paleotemperature history of the Cenozoic and the initiation of Antarctic glaciation: oxygen and carbon isotope analyses in DSDP Sites 277, 279, and 281. In Kennett, J.P., Houtz, R.E., et al., Init. Repts. DSDP, 29: Washington (U.S. Govt. Printing Office), 743-755.

Shipboard Scientific Party, 1995a. Site 926. In Curry, W.B., Shackleton, N.J., Richter, C., et al., Proc. ODP, Init. Repts., 154: College Station, TX (Ocean Drilling Program), 153-232.

, 1995b. Site 929. In Curry, W.B., Shackleton, N.J., Richter, C., et al., Proc. ODP, Init. Repts., 154: College Station, TX (Ocean Drilling Program), 337-417.

Vincent, E., and Berger, W.H., 1985. Carbon dioxide and polar cooling in the Miocene: the Monterey Hypothesis. In Sundquist, E.T., and Broecker, W.S. (Eds.), The Carbon Cycle and Atmospheric $\mathrm{CO}_{2}$ : Natural Variations Archean to Present. Geophys. Monogr., Am. Geophys. Union, 32:455-468.

Woodruff, F., and Savin, S.M., 1989. Miocene deepwater oceanography. Paleoceanography, 4:87-140.

, S.M., 1991. Mid-Miocene isotope stratigraphy in the deep-sea: high resolution correlations, paleoclimatic cycles, and sediment preservation. Paleoceanography, 6:755-806.

Woodruff, F., Savin, S.M., and Douglas, R.G., 1980. Biological fractionation of oxygen and carbon isotopes by Recent benthic foraminifera. Mar. Micropaleontol., 5:3-11.

Wright, J.D., and Miller, K.G., 1992. Miocene stable isotope stratigraphy, Site 747, Kerguelen Plateau. In Wise, S.W., Jr., Schlich, R., et al., Proc. ODP, Sci. Results, 120: College Station, TX (Ocean Drilling Program), 855-866.

- 1993. Southern Ocean influences on late Eocene to Miocene deepwater circulation. In Kennett, J.P., and Warnke, D.A. (Eds.), The Antarctic Paleoenvironment: A Perspective on Global Change, Part II. Antarct. Res. Ser., 60:1-25.

Wright, J.D., Miller, K.G., and Fairbanks, R.G., 1992. Early and middle Miocene stable isotopes: implications for deepwater circulation and climate. Paleoceanography, 7:357-389.

Yilmaz, Y., 1993. New evidence and model on the evolution of the southeast Anatolian orogen. Geol. Soc. Am. Bull., 105:251-271.

Zachos, J.C., Rea, D.K., Seto, K., Niitsuma, N., and Nomura, R., 1992. Paleogene and early Neogene deep water history of the Indian Ocean: inferences from stable isotopic records. In Duncan, R.A., Rea, D.K., Kidd, R.B., von Rad, U., and Weissel, J.K. (Eds.), The Indian Ocean: A Synthesis of Results from the Ocean Drilling Program. Am. Geophys. Union, Geophys. Monogr., 70:351-386.

Ms 154SR-142

Date of initial receipt: 4 December 1995

Date of acceptance: 21 April 1996 